\title{
छூ \\ Operational performance of the CERN injector complex with transversely split beams
}

\author{
S. Abernethy, ${ }^{1,2}$ A. Akroh, ${ }^{2}$ H. Bartosik, ${ }^{2}$ A. Blas, ${ }^{2}$ T. Bohl, ${ }^{2}$ S. Cettour-Cave, ${ }^{2}$ K. Cornelis, ${ }^{2}$ \\ H. Damerau, ${ }^{2}$ S. Gilardoni, ${ }^{2}$ M. Giovannozzi, ${ }^{2, *}$ C. Hernalsteens, ${ }^{2,3}$ A. Huschauer, ${ }^{2,4}$ V. Kain, ${ }^{2}$ \\ D. Manglunki, ${ }^{2}$ G. Métral, ${ }^{2}$ B. Mikulec, ${ }^{2}$ B. Salvant, ${ }^{2}$ J.-L. Sanchez Alvarez, ${ }^{2}$ R. Steerenberg, ${ }^{2}$ \\ G. Sterbini, ${ }^{2}$ and $\mathrm{Y} . \mathrm{Wu}^{2}$ \\ ${ }^{1}$ Department of Physics, Engineering Physics and Astronomy, 64 Bader Lane, Queen's University, \\ Kingston K7L 3N6, Canada \\ ${ }^{2}$ CERN Beams Department, CH 1211 Geneva 23, Switzerland \\ ${ }^{3}$ École Polytechnique Fédérale de Lausanne, LPAP, CH-1015 Lausanne, Switzerland \\ ${ }^{4}$ Vienna University of Technology, Karlsplatz 13, AT-1040 Vienna, Austria
}

(Received 3 November 2016; published 3 January 2017)

\begin{abstract}
With the progress made in 2015, the beams produced by the CERN Proton Synchrotron using multiturn extraction (MTE) have been delivered to the Super Proton Synchrotron (SPS) for the fixed-target physics run. Operation successfully started in the second half of September 2015 and continued until the end of the proton physics program by mid November. In this paper the overall performance and beam quality is discussed in detail considering the complete chain of accelerators, from the PS-Booster to the SPS. Moreover, a thorough comparison of the global performance of the MTE scheme against the previously used technique, the so-called continuous transfer (CT), is also carried out.
\end{abstract}

DOI: 10.1103/PhysRevAccelBeams.20.014001

\section{INTRODUCTION}

Since the 1970s, the proton beam delivered by the CERN Proton Synchrotron (PS) to the Super Proton Synchrotron (SPS) for fixed-target physics has been based on the socalled continuous transfer (CT) [1,2]. This peculiar extraction mode was implemented to extract the proton beam over five PS turns, so that with two subsequent extractions from the PS, ten elevenths of the SPS circumference can be filled. With this approach, the filling time, and hence the proton flux on the SPS experimental area targets, was optimized. In the PS, prior to extraction, the beam is debunched and the horizontal tune is set to 6.25 to rotate the beam by $90^{\circ}$ in phase space each machine revolution. A complex system of slow and fast bumps is then switched on, so that the beam is sent on the thin foil of an electrostatic septum, which effectively shaves a part of the beam off, and by means of a second magnetic septum it is extracted out of the PS ring.

The ever-increasing requests for protons on target [3] imposed a critical review and an improvement of the beam loss management in the PS. In fact, detailed analysis showed a number of drawbacks of the CT, namely high

\footnotetext{
*Corresponding author. massimo.giovannozzi@cern.ch

Published by the American Physical Society under the terms of the Creative Commons Attribution 4.0 International license. Further distribution of this work must maintain attribution to the author(s) and the published article's title, journal citation, and DOI.
}

losses over a large fraction of the machine circumference, due to the particles scattered off the foil of the electrostatic septum [4], and poor betatron matching in the SPS [5,6]. This called for an in-depth review of the extraction process in the PS aiming at a drastic reduction of beam losses.

The alternative extraction process was proposed in 2002 [7] and is based on a novel beam manipulation in which the beam distribution is split in the horizontal phase space by means of stable islands generated by non-linear magnetic fields (sextupoles and octupoles), which are moved adiabatically. The split beam can then be extracted over several turns by means of slow and fast bumps, and a single magnetic septum. The expected gain with respect to CT is lower levels of beam losses well localized in the accelerator circumference. The whole process, beam splitting and extraction, has been named multiturn extraction (MTE). To note that in the rest of the paper the beam splitting will be also referred to as magnetic or transverse splitting, while beam slicing or shaving refers to the manipulation performed for the CT. The initial idea was studied by means of numerical simulations together with beam measurements, using the hardware available in the PS at the time [8-15]. Based on the successful outcome of these studies, an indepth analysis aiming at the implementation of the proposed scheme in the PS, in view of making MTE the operational replacement of CT, was launched in 2006 [16].

The hardware was designed and successfully commissioned in 2008 and the beam commissioning started shortly thereafter [17-19]. A first short operational period in 2010 was essential to gather experience with the novel technique. 
In spite of an encouraging confirmation of the design choices, in particular showing the feasibility of the transverse splitting, a number of issues were observed: first, the transverse splitting and the injection trajectories into the SPS were suffering from poor reproducibility; second, an increased radiation level in the extraction region of the PS was observed. The latter was due to the longitudinal structure of the beam, which had to be transferred debunched to the SPS. The beam losses occurring during the rise time of the extraction kickers were not acceptable because, albeit small, were localized on the extraction septum. The fact that this issue only occurs for MTE but not for CT beams is based on the different extraction approaches, as the longitudinal structure is identical in both cases. In fact, in the CT case the kickers are pushing the beam on an electrostatic septum and the sliced beam is separated from the circulating one. In the downstream extraction line this slice then enters the magnetic septum to experience the final deflection into the transfer line. On the other hand, in the MTE case the beam is swept, during the kickers rise time, over the magnetic septum directly, which results in a much higher level of losses at the location of this device.

The solution to the excessive radiation levels in the PS extraction area was found by introducing a so-called dummy septum [20-24] in the extraction scheme. This device is installed $8 \mathrm{~m}$ upstream of the magnetic septum and contains a blade, which is supposed to intercept the protons during the rise time of the extraction kickers to avoid an interaction with the magnetic septum. The principle consists of creating a single point of losses on a passive device, which is well-shielded by an appropriate concrete enclosure. Albeit simple in principle, this solution implied a deep revision of all the single-turn extraction schemes, as the dummy septum is effectively a horizontal aperture restriction that should be avoided by all extracted beams, except the MTE one. Having solved this issue, the lack of reproducibility of the transverse splitting and of the injection trajectories into the SPS was still preventing the operational deployment of MTE. After a long search, at the beginning of 2015, a nonreproducible ripple in the power converters of some special coils in the PS main magnets was clearly identified as being well correlated with the fluctuations of the properties of the split beam $[25,26]$. This opened again the possibility of delivering the MTE beam to the SPS in the second part of the 2015 run and since September 21st, 2015 the CT extraction has been officially replaced by the MTE scheme. As far as the fluctuations of the PS extraction trajectories are concerned, these are partly due to the same ripple of the power converters that are affecting the transverse splitting as well as to hysteresis effects due to the change between different magnetic cycles.

The operational period in 2015 gathered experience and interesting beam data that can be used to perform a detailed and quantitative analysis of the performance of the MTE beam in the PS and the SPS, as well as a comparative analysis with CT. It is worth stressing that, although the source of the nonreproducibility of the MTE beam has been identified, the implemented mitigation measures have not completely solved the problem. Therefore, time variation of the MTE beam properties are still present and the analysis presented in this paper aims also at determining correlations between various beam parameters in order to identify the key observables and provide specifications to ensure optimal performance of the whole injector complex.

The structure of the paper is the following: in Sec. II the roles of each circular machine in the chain of accelerators, namely PS-Booster (II A), PS (II B), and SPS (II C) are reviewed, discussing the several activities aimed at commissioning MTE or at improving its performance. In Sec. III the detailed analysis of the overall performance of the MTE beams is carried out and discussed. This analysis is split into different parts, referring to either the analysis of the extraction performance, hence focusing on the PS (III A), or to the analysis of injection efficiency and beam behavior in the SPS cycle up to extraction (III B). A detailed comparison between CT and MTE is performed in Section III D, while conclusions are drawn in Sec. IV. In Appendix A the properties of each figure-of-merit used in the performance analysis and their mutual relationships are presented, while in Appendix B the harmonic analysis of the intensity spill for MTE and CT beams is discussed, and finally in Appendix $\mathrm{C}$ the application of the concepts developed in previous appendices to the measured data is reported.

\section{OVERVIEW OF THE INJECTOR CHAIN FOR MTE BEAMS}

\section{A. PS-Booster}

The PS-Booster (PSB) is the first circular machine in the CERN proton injector chain. The lattice is based on separate function magnets and triplets' focusing, with a transition energy of $3.89 \mathrm{GeV}$ [27]. The beam is injected at $50 \mathrm{MeV}$ kinetic energy from the proton linear accelerator Linac 2. The PSB accelerates various beams for the LHC and for fixed-target experiments, featuring extracted intensities varying over more than three orders of magnitude. At these low energies, one of the main performance limitations consists of transverse emittance growth due to direct space charge. The PSB was constructed in 1972 exactly to mitigate this limitation at injection energy in the downstream accelerator, the PS. It features four rings stacked on top of each other to be able to divide the full beam intensity by four, accelerate the four beam fractions and recombine them again in the transfer line after extraction at $1.4 \mathrm{GeV}$ kinetic energy. Injection occurs at nonzero magnetic field variation to leave the high space charge regime as quickly as possible. 
Together with the Linac 2, which provides initial transverse emittance around $1 \mu \mathrm{m}$ in both planes, the PSB basically defines the transverse emittances of all proton beams produced at CERN. This is related to the multiturn injection process, where the Linac 2 beam is injected at the location of a magnetic septum over a certain number of PSB turns on a decaying-amplitude injection bump. Therefore, the injected-beam trajectory steadily approaches the circulating-beam orbit, as well as the septum blade. While this allows accumulating more than $10^{13}$ protons/ring, it also leads to injection losses at the septum blade that usually exceed $40 \%$ of the injected intensity. It is worth noting that the injection process naturally induces a linear relationship between injected intensity and transverse beam emittances.

The MTE beam should be delivered to the SPS fixedtarget experiments providing an intensity varying over a certain range. The SPS is particularly sensitive to the value of the vertical beam emittance (see Sec. II C) due to the limited vertical aperture. Therefore, it was of prime importance to minimize the vertical emittance of the MTE beam in the PSB for all intensities needed. The task was particularly difficult for higher intensities, as transverse emittances are larger because of the multiturn injection process and the large space charge tune spread. In addition, the horizontal emittance is supposed to be larger than the vertical one for improving the transverse splitting in the PS. All these requirements are difficult to achieve with the present injection scheme, as transverse phase space painting will become available only after the connection of the Linac $4[28,29]$ and the new $H^{-}$injection scheme into PSB. Hence, the following parameters had to be fine-tuned to fulfill the multiple constraints: (i) Number of injected turns (ii) Angle and position of the injected beam through orthogonal knobs (iii) Amplitude of the injection bump (iv) Injection time on the decaying part of the injection bump (v) Optimization of the dynamic evolution of the working point (vi) Appropriate resonance compensation, e.g., for the half-integer resonance.

Particular attention had to be paid to the optimization of the working point along the PSB magnetic cycle. Trim power supplies are available in each PSB ring for the main focusing and defocusing quadrupoles to modify the ring's bare tune, i.e., the tune for a zero-intensity beam. For highintensity beams, the direct space charge vertical tune spread at PSB injection is very large ( $>0.7$ [30]), and therefore the bare working point has to be increased to avoid losses at the integer resonance, which is usually done during the first $200 \mathrm{~ms}$ after injection (note in the PSB the total duration of acceleration is $530 \mathrm{~ms}$ ). For the CT beam, the vertical working point at injection was set just below the halfinteger (the vertical tune spread for an extracted intensity of $600 \times 10^{10}$ protons/ring $>0.5$ [30]), reaching vertical emittance values at extraction just below $5.5 \mu \mathrm{m}(1 \sigma$ normalized). Unfortunately, it was impossible to obtain the small vertical emittance goal of $\approx 4 \mu \mathrm{m}$ for the MTE beam using the same dynamic working point. Moreover, it should also be mentioned that toward the second half of 2015 the beam current delivered by Linac 2 was lowered by about $15 \%$ compared to previous years, which resulted in an increased number of injected turns that reached the same intensity, thus producing beams with larger transverse emittances.

The working point was optimized throughout the whole acceleration cycle until extraction with minimum linear coupling between the horizontal and vertical planes. The vertical working point at injection was increased for all rings to values between 4.65 and 4.7 (see Fig. 1) as well as the horizontal one from 4.25 to $\approx 4.31$. As can be seen from Fig. 1, and considering also the large Laslett tune spread, many resonances are crossed during the cycle. Obviously, the harmful resonances have to be well compensated to avoid losses, which is accomplished by means of a set of multipolar magnets available in the PSB rings. Nevertheless, the choice of the vertical tune above the vertical half-integer resonance line leads to some losses, which are nevertheless at low energy. However, given that mainly large-amplitude particles are lost, this results in a decrease in vertical emittance, which is a positive effect. It is worthwhile noting that the total amount of losses along the cycle was still comparable between CT and MTE beams.

The transverse emittance values that could be achieved in the PSB at extraction energy for the corresponding total MTE intensities are summarized in Table I.

In Fig. 2, the impact of the special setup applied to the MTE beams in the PSB is shown. The transverse emittances are plotted as a function of the bunch intensity. Two

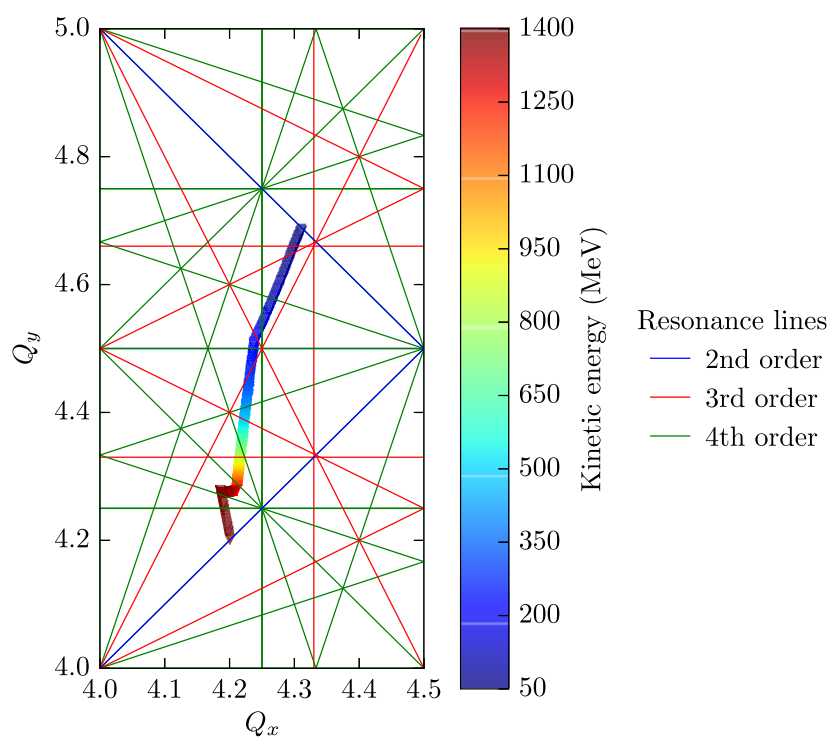

FIG. 1. Calculated zero-intensity working point evolution along the PSB cycle for the MTE beam. Injection takes place in the upper right end of the working point lines. Resonance lines up to 4th order are shown. 
TABLE I. Measured average normalized emittance values for the MTE beam and different total intensities, expressed in protons per pulse (ppp). The emittance is expressed as $1 \sigma$ normalized value.

\begin{tabular}{lcc}
\hline \hline Total intensity $\left[10^{10} \mathrm{ppp}\right]$ & $\left\langle\epsilon_{x}\right\rangle[\mu \mathrm{m}]$ & $\left\langle\epsilon_{y}\right\rangle[\mu \mathrm{m}]$ \\
\hline 1500 & 5.2 & 3.4 \\
1800 & 6.1 & 3.7 \\
2000 & 6.8 & 4.2 \\
\hline \hline
\end{tabular}

cases are shown: one corresponding to the standard cycle configuration (blue) and one for the optimized cycle (red). In both cases the linear dependence of emittances on intensity is clearly visible. On the other hand, a clear difference is seen for the evolution of the vertical emittance. Indeed, not only has the offset term of the linear relations been decreased, but also the slope, thus making the vertical emittance for the optimized cycle smaller and also less dependent on the intensity. This was certainly a major achievement that allowed us to test high-intensity MTE beams in the SPS.

In the longitudinal plane, maximum radio-frequency (rf) voltages of both the $h=1$ and $h=2$ PSB cavities, the latter shifted by $180^{\circ}$ in phase, are applied at injection to generate the largest possible rf bucket area, in order to maximize capture efficiency and to minimize space charge effects by flattening the longitudinal distribution. At extraction, the requirements for the MTE beam are
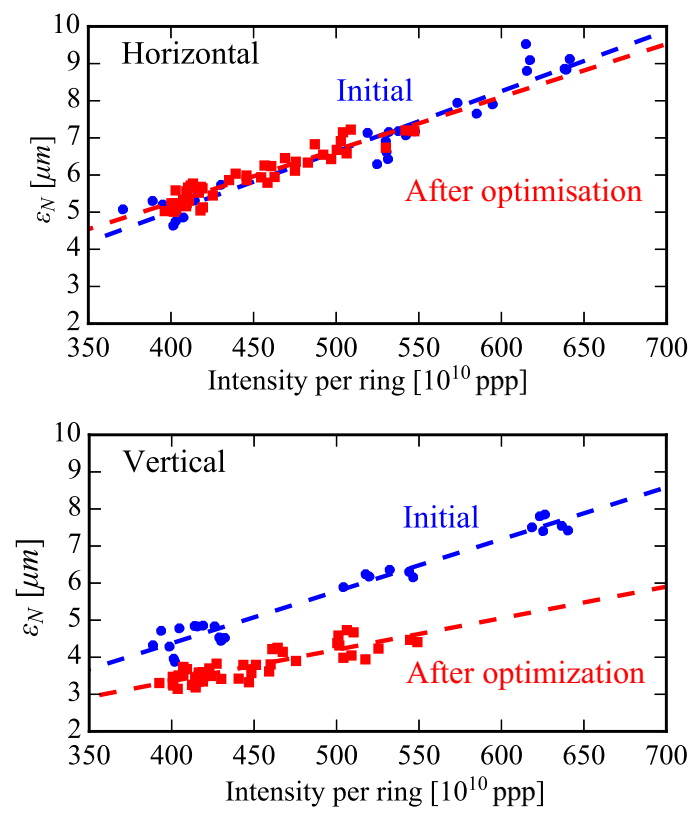

FIG. 2. Measured transverse normalized emittances in the PSB as a function of bunch intensity. The situation for the standard setup ("Initial") as well as the special one ("After optimization") is shown and proves the reduced dependence on intensity of the vertical emittances for the special configuration. The emittance is expressed as $1 \sigma$ normalized value.

defined by the PS. Injection into the PS is performed with a single-batch, bunch-to-bucket transfer at $h=8$, which imposes that longitudinal bunch splitting is performed in each of the four PSB rings, according to a well-established operational beam manipulation. The full bunch length at extraction is around $160 \mathrm{~ns}$ and the longitudinal emittance (matched area) is $1.3 \mathrm{eVs}$. After synchronization, the two bunches per ring are recombined after extraction and then transferred to the PS ring.

\section{B. Proton synchrotron}

The PS is the oldest CERN synchrotron [31], with a lattice based on combined function magnets and doublets focusing, with a transition energy of $5.72 \mathrm{GeV}$. Being at the heart of the injector chain, the PS is essential for the definition of the key parameters of the beams delivered to the SPS and to the LHC, see Ref. [32] for an overview of the several beam manipulations performed in the PS ring, while a summary of main parameters is listed in Table II.

Its role is essential for the production of proton beams based on transverse splitting, a manipulation performed on the flat top of the magnetic cycle at the extraction momentum of $14 \mathrm{GeV} / c$. Even if this section focuses at the beam manipulation at flat top, it is worth mentioning some key features of the low-energy part of the PS cycle, which

TABLE II. Basic parameters of the PS ring. The cycle length refers to the time needed from injection up to extraction energy (including the time for single-, multi-turn, or slow beam extraction) and back again to injection. Such a time is multiple of the PSB repetition rate, which is $1.2 \mathrm{~s}$.

Accelerated particles

Injection kinetic energy $[\mathrm{GeV}]$

Maximum energy [GeV]

Circumference [m]

Magnetic lattice focusing

Focusing type

Number of main magnets

Bending magnetic field $[\mathrm{T}]$

Betatron oscillations/turn

Minimum and maximum

$\beta$ function [m]

Minimum and maximum

Dispersion function [m]

Transition energy [GeV]

Magnetic cycle length [s]

Straight sections

Main rf system (tunable)

Auxiliary rf systems [MHz] $\mathrm{p}$ and several types of ions 1.4

26

$200 \pi$

Alternating-gradient, combined-function FOFDOD

100

0.101 at injection, 1.24 at $26 \mathrm{GeV}$ $6.25(\mathrm{~h}), 6.25$ (v)

$11.7-22.6$

$2.3-3.1$

5.72

1.2 (up to $20 \mathrm{GeV}$ )

2.4 (up to $26 \mathrm{GeV}$ )

number $=100,80$ of $1.6 \mathrm{~m}, 20$ of $3 \mathrm{~m}$

$10+1$ cavities,

2.6-9.5 MHz, $200 \mathrm{kV}$ total maximum

$13.3,20,40,80,200$ 
are relevant for the beam splitting at top energy: (i) A reduced linear coupling, achieved by means of two families of skew quadrupoles distributed along the PS ring, aimed at preserving the special emittance ratio. (ii) A longitudinal splitting (see Fig. 3, upper) changing the harmonic number from $h=8$ (used at injection and in the low-energy part of the acceleration) to $h=16$ (used during most of the acceleration and at flat top). The description of the details of the transverse splitting can be found in Refs. [25,26]. Here, only the main features will be outlined.

The transverse splitting and transport process, which efficiently replaces the slicing performed with $\mathrm{CT}$, occurs prior to and prepares for beam extraction.

The time variation of the dedicated sextupoles and octupoles used to trap particles and of the rf voltage is shown in Fig. 3 (upper), while in the lower part the variation of the key physical parameters is reported, assuming the following notation:

$$
\begin{aligned}
& Q_{x}\left(J_{x}, J_{y}, \delta\right)=Q_{x}+2 h_{2,0} J_{x}+h_{1,1} J_{y}+Q_{x}^{\prime} \delta+\frac{1}{2} Q_{x}^{\prime \prime} \delta^{2} \ldots \\
& Q_{y}\left(J_{x}, J_{y}, \delta\right)=Q_{y}+h_{1,1} J_{x}+2 h_{0,2} J_{y}+Q_{y}^{\prime} \delta+\frac{1}{2} Q_{y}^{\prime \prime} \delta^{2} \ldots
\end{aligned}
$$

where $\delta$ and $J_{x}, J_{y}$ are the relative momentum offset and the actions in the horizontal and vertical plane. The terms
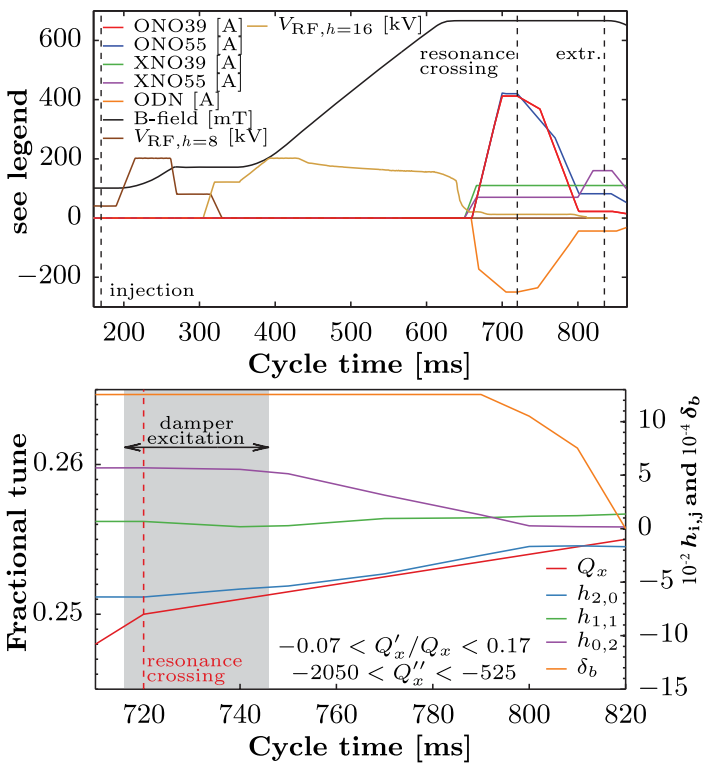

FIG. 3. Upper: Time evolution of the strength of the key families of sextupoles, octupoles, and of rf voltage during the resonance crossing process. Bottom: The variation of the corresponding main observables of beam dynamics is also shown. $\delta_{b}$ is the bucket height and is equal to $3 \sigma_{\delta}$, with $\sigma_{\delta}$ the rms beam momentum spread. The location of the main elements in the PS ring is shown in Fig. 5. The elements with name starting with $\mathrm{X}$ or $\mathrm{O}$ are sextupoles, or octupoles, respectively. $h_{2,0}, h_{0,2}, h_{1,1}$ represent the detuning due to nonlinear motion in the horizontal, vertical plane, and the nonlinear coupling between them [33]. $Q_{x, y}^{(n)}$ are the $n$th order chromaticities. The coefficients appearing in Eqs. (1) are well-known functions of the strength of quadrupoles, sextupoles, and octupoles in the PS ring [33].

Sextupole and octupole magnets are used to generate the stable islands and to control their parameters, size and position, to maximize the trapping probability. The coupling between horizontal and vertical motion, which could negatively affect the overall trapping efficiency, is reduced by minimizing $h_{1,1}$ and the vertical emittance. In theory, the coefficients $Q_{x}^{(n)}$ should be minimized to reduce the coupling between transverse and longitudinal motion. In practice, due a limited number of correcting circuits, only $Q_{x}^{\prime}$ is controlled, the other parameters being left free. Similarly, in the vertical plane the variation of the tune is left free together with the other chromatic parameters $Q_{y}^{(n)}$.

The impact of the nonlinear coupling between the two transverse planes has been probed by measuring, by means of a wire scanner, the vertical emittance at PS injection and just prior to extraction, i.e., after beam splitting. In Fig. 4 the $1 \sigma$ normalized emittances are shown as a function of the beam intensity. For each intensity value, roughly ten emittance measurements have been performed and the values shown in the plot are the mean of each series. The spread is completely negligible on that scale, implying a very good reproducibility of the measurement and of the beam parameters. It is also worth stressing that the accuracy with which the emittance is derived from wire scanner measurements in the PS ring is estimated to be of the order of 5\% [34]. At injection, the comparison between the emittance measured in the PS and the corresponding value from the fit of PSB values (as shown in Fig. 2) is very good: the difference is almost constant over the intensity range

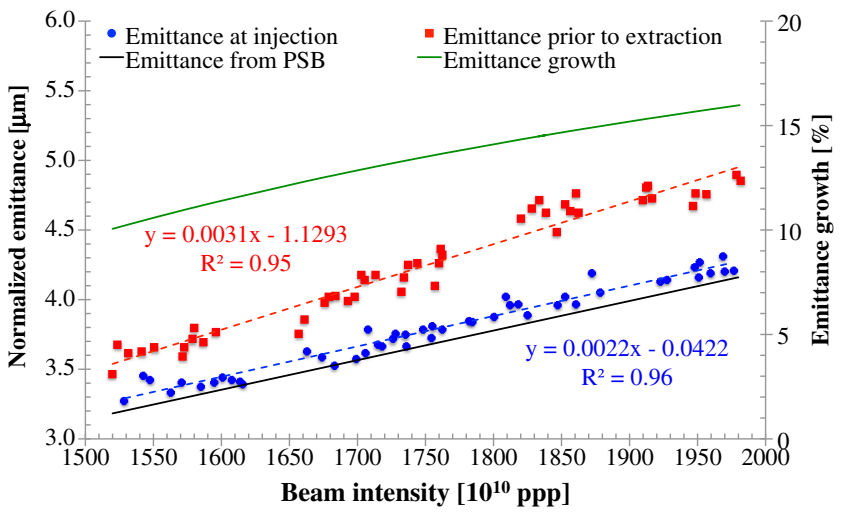

FIG. 4. Normalized $1 \sigma$ vertical emittance in the PS ring at injection and after beam splitting as a function of total beam intensity. The relative emittance growth is also shown together with the measured emittance evolution vs intensity out of the PSB (see Fig. 2). 
shown in Fig. 4 and less than 3\%. The measured emittances at injection and at $14 \mathrm{GeV} / c$ feature an offset and a slightly different slope as a function of beam intensity, which points to some emittance growth. The value is in the range $10 \%$ to $16 \%$, but it should be noted that the observed emittance growth represents an upper bound of the increase, possibly generated by nonlinear coupling during beam splitting. In fact, some of the emittance increase could be generated during acceleration and the ramp up of sextupoles and octupoles while preparing for the splitting.

Figure 5 shows the linear optical parameters of the PS ring together with the location of the key elements used for MTE (upper part) together with the beam envelopes of the two beams after splitting and before extraction takes place (lower part). The large occupancy of the PS ring mechanical aperture due to the split beam is clearly visible, where the beam envelopes are reported as $\sqrt{9 \sigma_{\beta}+4 \sigma_{\delta}}$ and $\sigma_{\beta}$ stands for the betatronic beam size.

To perform the splitting, a horizontal 4 th order resonance is crossed by changing the horizontal tune while keeping the vertical one constant. This variation is obtained by means of two families of quadrupoles that are distributed along the PS circumference. For $30 \mathrm{~ms}$ (one PS turn corresponds to $2.1 \mu \mathrm{s}$ ) after resonance crossing, the beam is excited horizontally by a wide band stripline kicker, used to provide an excitation in open loop at a frequency close to
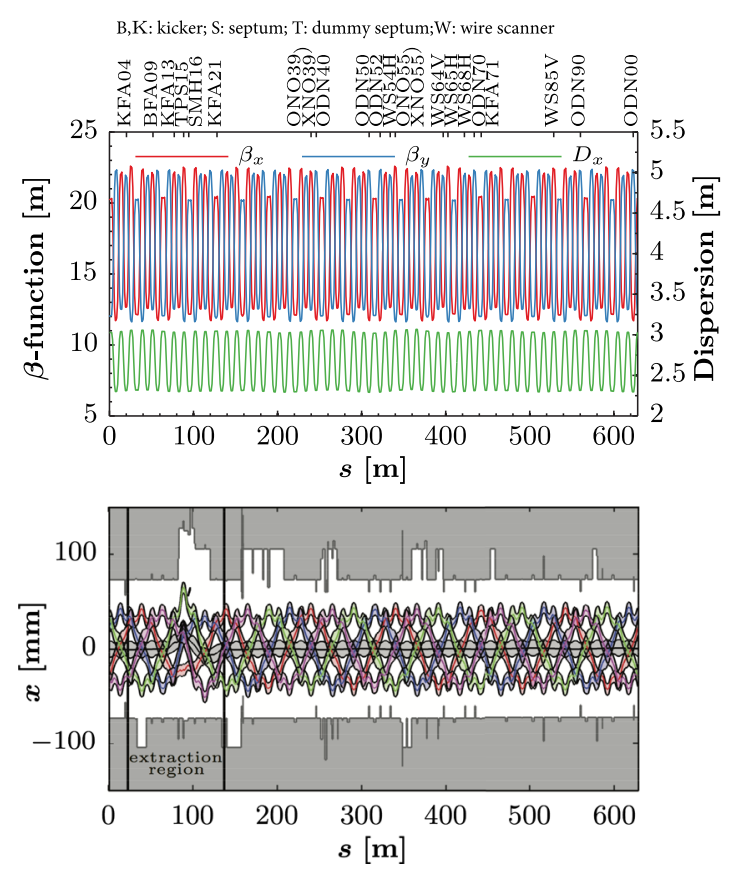

FIG. 5. Upper: Evolution along the PS circumference of the lattice functions, showing also the location of the essential elements for MTE. Bottom: Horizontal ring aperture together with beamlets and core envelope just prior to the pulsing of the kickers. The extraction region indicates the section of the ring where orbit bumps are present. The element name includes the number of the straight section in which the device is installed. the resonant tune frequency. This has shown to improve the trapping probability into the stable islands. After splitting, the beam is made of two disconnected parts: the beamlets in the stable islands, which form a structure four machine turns long and the beam core. This core is made of the particles left around the center of phase space after resonance crossing and is only one machine-turn long. Beam extraction proper is made by a set of fast dipoles (also called kickers) generating a closed orbit deformation lasting five machine turns.

As far as the longitudinal dynamics is concerned, the rf voltage, $V_{\text {rf }}$, of the $10 \mathrm{MHz}$ main cavity system is reduced before the resonance-crossing stage to minimize even further the coupling between the transverse and longitudinal degrees of freedom by reducing $\delta$ [see Eq. (1)]. At the end of the splitting process, $V_{\text {rf }}$ is set to zero to perform a complete debunching of the beam from the original $h=16$ configuration. The debunching could not be performed before beam splitting as a precise control of the beam radial position is essential for the optimal control of the resonance-crossing process. Prior to extraction a partial recapture using a $200 \mathrm{MHz}$ system is performed and the continuous beam, with an intensity modulation, is ready for transfer to the SPS, where it will be recaptured using the $200 \mathrm{MHz}$ main rf system.

As mentioned earlier, the overall performance of the splitting process is very sensitive to the magnetic status of the PS ring magnets. Following intense studies and beam tests, it turned out that the main source of nonreproducibility of the PS transverse splitting gymnastics is linked to a series of special coils, the so-called pole-face-windings (PFW) and the figure-of-eight loop (F8L) installed in the combinedfunction main magnets, which are used to control the PS working point, i.e., $Q_{x, y}$ and $Q_{x, y}^{\prime}$ (see Ref. [32] and references therein, as well as the sketch in Fig. 6).

In fact, the switch-mode power converters of these coils operate at $5 \mathrm{kHz}$ (for the PFWs) and $2.5 \mathrm{kHz}$ (for the F8L), and generate a current ripple at that frequency. Moreover, the amplitude of such a component turned out to be larger than expected and not constant in time. The latter issue is a consequence of the lack of synchronization of the control clocks of the power converters of the different circuits. The main effect of such a ripple is to change the machine parameters and hence to alter the overall efficiency of the MTE process, as is shown in Fig. 7.

The figure-of-merit of the MTE performance [25] is

$$
\text { MTE efficiency }=\eta_{\mathrm{MTE}}=\frac{\left\langle I_{\text {Islands }}\right\rangle}{I_{\text {Total }}},
$$

where $\left\langle I_{\text {Islands }}\right\rangle$ and $I_{\text {Total }}$ stand for the average intensity in the islands and the total beam intensity, respectively. The nominal efficiency is $20 \%$, corresponding to an equal beam sharing between islands and core, with a minimum acceptable value of $19 \%$ set by the SPS. 

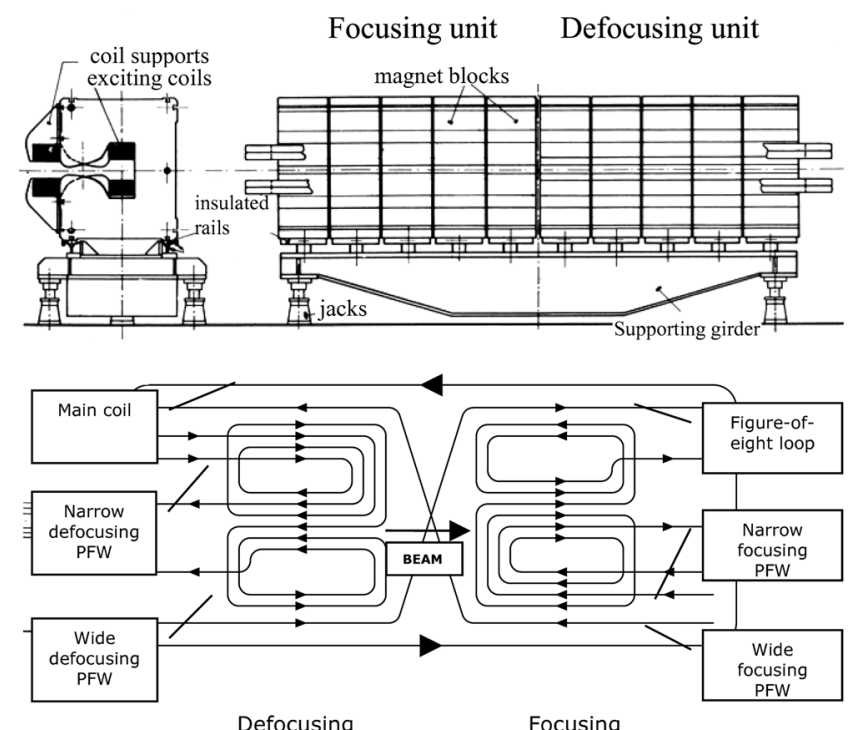

Defocusing

Focusing

FIG. 6. Sketch of the PS main magnet (upper) and of the five circuits used to control tunes and chromaticities (lower), from Ref. [35]. The additional coils enable generating a transverse variation of the magnetic field, which is used to produce nonlinear field components essential for the control of the transverse splitting.

In general, if no particular symmetry is assumed for the extracted spill intensity, then the following figure-of-merit

$$
\eta_{\mathrm{DC}}=\frac{1}{T} \frac{\left[\int_{0}^{T} I(t) d t\right]^{2}}{\int_{0}^{T} I^{2}(t) d t}
$$

can be used, which represents the deviation of the extracted intensity from the case of a perfectly constant spill. In this case $I(t)$ represents the extracted intensity as a function of time and $T$ is the duration of the extracted spill. In the case of a multiturn extraction it is assumed that $T=n \tau$ where $\tau$ is the revolution period and $n$ is the number of extracted turns, which for our case, i.e., MTE or CT, corresponds to
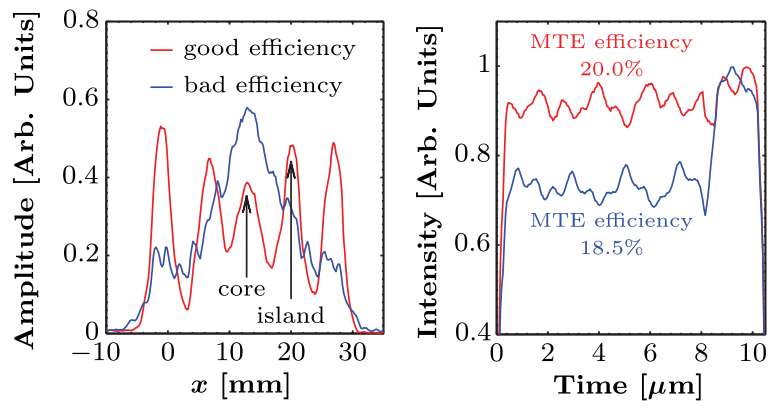

FIG. 7. Left: Measured horizontal beam distribution for different efficiency of the beam splitting. Right: Corresponding intensity profile measured by a beam current transformer in the transfer line between the PS and the SPS.

$n=5$. Some properties of $\eta_{\mathrm{MTE}}$ and $\eta_{\mathrm{DC}}$ are listed in Appendix A.

\section{Super Proton Synchrotron}

The SPS is the last accelerator of the CERN injector complex. It delivers a beam to the North Area fixed target experiments using a resonant slow extraction, serves as LHC injector, and provides beam to the HiradMat [36] and AWAKE [37,38] facilities. Between 2007 and 2012, the SPS provided high intensity fixed target beams for the CERN Neutrino to Gran Sasso (CNGS) experiment [3]. Furthermore, the SPS might deliver high intensity beams to the proposed future fixed target facility for the Search for HIdden Particles (SHIP) [39].

The SPS has a regular FODO lattice with six long straight sections (LSSs) [40], featuring a transition energy of $21.6 \mathrm{GeV}$ : a summary of main parameters are listed in Table III. The proton beams for fixed target physics are injected at a momentum of $14 \mathrm{GeV} / c$ and therefore need to cross transition in the early part of the acceleration cycle to the $400 \mathrm{GeV} / c$ extraction momentum. Due to the high intensity of the fixed-target beams used in routine operation, beam loss in the SPS has to be kept as low as possible to limit activation of the ring.

The shape of the vacuum chambers follows the beam size variation around the ring circumference and the aperture is optimized to accommodate the horizontal beam size that

TABLE III. Basic parameters of the SPS ring. The cycle length refers to the time needed from injection up to extraction energy (including the time for single-, multi-turn, or slow beam extraction) and back again to injection. Such a time is a multiple of the PSB repetition rate, which is $1.2 \mathrm{~s}$.

Accelerated particles

Injection energy [GeV]

Maximum energy [GeV]

Circumference [m]

Magnetic lattice focusing

Focusing type

Number of main magnets

Bending magnetic field [T]

Betatron oscillations/turn

Minimum and maximum

$\beta$ function [m]

Minimum and maximum

Dispersion function [m]

Transition energy [GeV]

Magnetic cycle length [s]

Long straight

sections

Main rf system

Auxiliary rf systems [MHz] $\mathrm{p}$ and several types of ions

14

450

$2200 \pi$

Alternating-gradient, separated-function

FODO

744 dipoles, 216 quadrupoles 0.06 at injection,

$2.03 \mathrm{~T}$ at $450 \mathrm{GeV}$

26.62 (h), 26.58 (v)

$20-107$

$-0.5-4.5$

21.6

10.8 (up to $400 \mathrm{GeV}$ )

number $=6$,

$130 \mathrm{~m}$ each

4 cavities, $200 \mathrm{MHz}$,

$7 \mathrm{MV}$ total maximum 

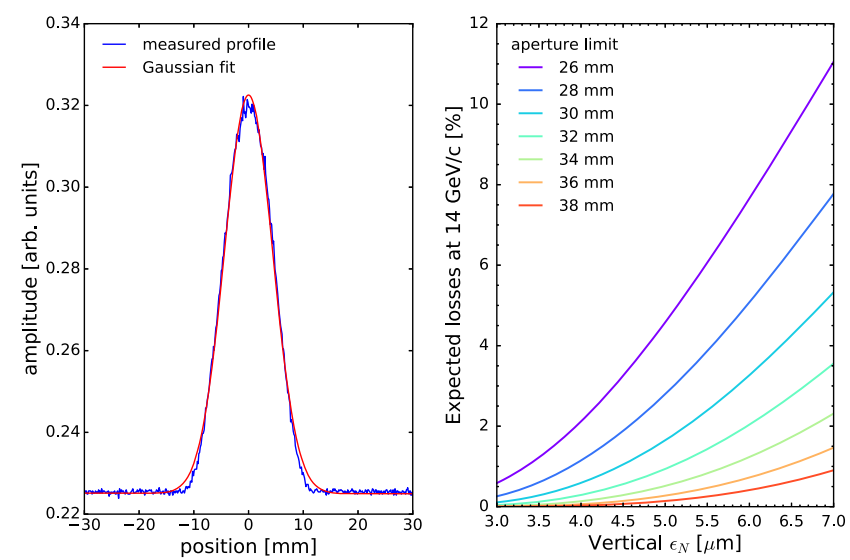

FIG. 8. Left: Wire scanner vertical profile measurement, showing the profile tails, cut by the aperture restrictions, leading to beam scraping and losses. The extent of the profile is hence a measure of the global available aperture. Right: Expected losses at the SPS flat bottom as a function of the vertical normalised emittance. The global aperture bottleneck is the internal dump, TIDVG.

grows larger due to the increased momentum spread during transition crossing. Due to the flat vacuum chambers in the dipole magnets, the vertical acceptance becomes critical at injection energy and in the first part of acceleration.

Figure 8 (left) shows the vertical beam profile of a fixed target beam measured with a SPS wire scanner at injection energy after excitation with the tune kicker, which is used as a procedure to probe the ring aperture. The sharp edges of the beam profile result from losses at aperture restrictions around the ring together with filamentation due to strong amplitude detuning induced by octupoles. The full width of the beam profile corresponds to the vertical aperture limit of the machine scaled to the beta function at the wire scanner location. Based on this information, the losses to be expected when injecting a beam with a Gaussian vertical particle distribution can be calculated as a function of the vertical emittance, as shown in Fig. 8 (right), where different values of the aperture limits are considered. The aperture limit is scaled to the beta function at the TIDVG internal beam dump, which is by design the theoretical vertical aperture restriction of the whole machine.

The vertical acceptance of the SPS varies from year to year due to several reasons, such as, e.g., changes in the machine alignment due to magnet replacements. Measurements of the SPS aperture limit are performed routinely each year during the commissioning period so that possible degradation can be identified and, if possible, aperture bottlenecks removed. Figure 9 shows the evolution of the measured vertical aperture in the SPS using the excitation with the tune kicker and wire scanner measurement as described above. The vertical aperture limit measured at the wire scanner locations and scaled to the position of the internal beam dump TIDVG performed

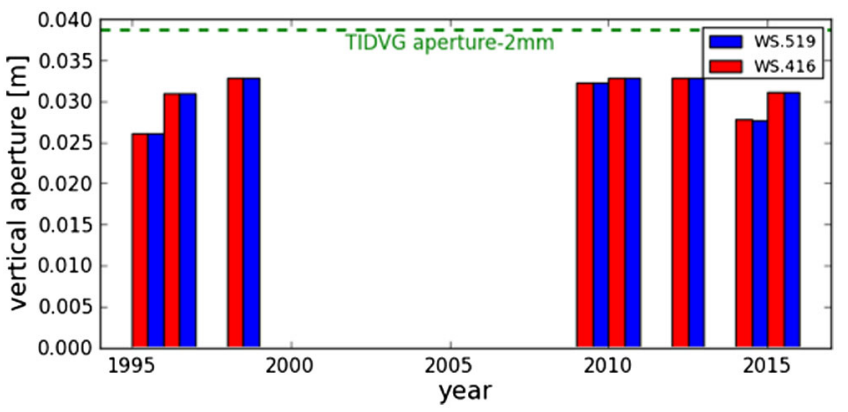

FIG. 9. Evolution of the measured SPS vertical aperture from 1995 to 2015. The extent of the transverse particle distribution of a blown up beam is evaluated with wire scanners as a measure of the global aperture. The measurements with two wire scanners are compared and translated to the location of the known SPS aperture bottleneck TIDVG.

between 1995 and 2015 range from 26 to $33 \mathrm{~mm}$, compared to the TIDVG aperture of $\approx 40 \mathrm{~mm}$. In the last years the vertical aperture was typically $32.5 \mathrm{~mm}$, but in 2015 after long shutdown 1, the aperture was found to be reduced to $31 \mathrm{~mm}$.

The characteristics of the vertical beam emittance at SPS injection depend on the extraction scheme in the PS. The CT scheme profits from the emittance exchange optics [41] in the TT2-TT10 transfer line between the PS and the SPS so that the vertical emittance at SPS injection is defined by the horizontal beam emittance after the slicing at the electrostatic extraction septum in the PS. In the case of the MTE scheme, the emittance exchange is not applied, because the cycle-to-cycle fluctuation of the horizontal extraction trajectories at the PS would be transferred to the vertical plane at SPS injection, thus reducing even further the already limited vertical aperture. Therefore, for typical fixed target beam parameters at PS flat-top prior to extraction, the CT scheme provides smaller vertical emittance. Nevertheless, special measures were put in place in the beam production at the PSB (cf. Sec. II A), which allowed reducing the vertical emittance for the MTE beam so that similar values as with the CT scheme could be achieved in the last part of the 2015 run, i.e., about $4 \mu \mathrm{m}$ for an intensity of $1800 \times 10^{10} \mathrm{p}$ at PS extraction.

Apart from small vertical emittance, an important parameter for minimizing losses in the SPS is the amount of $200 \mathrm{MHz}$ structure on the beam extracted from the PS. In fact, the rf loops rely on this feature to work properly and to ensure good capture efficiency. Furthermore, the SPS requires an intensity difference not exceeding about 5\% between the five turns extracted from the PS, i.e., in the case of MTE, $\eta_{\mathrm{MTE}}=20 \% \pm 1 \%$, in order to guarantee a regular spill structure during the resonant slow extraction to the experiments.

The work on the commissioning of the MTE beam in the SPS in 2015 started with machine development studies aimed at optimizing the transfer line steering of TT10, 
performing a careful correction of the closed orbit at injection energy, and of the energy matching between the PS and the SPS. The latter is more delicate with MTE than $\mathrm{CT}$ beams, due to reduced freedom in setting the radial beam position at PS extraction. In fact, depending on the value of the radial position, the feed down from the nonlinear magnetic components in the PS main magnets changes, thus varying the performance of the transverse splitting in the PS. Another difficulty at the beginning of the MTE operation in the SPS came from the nonuniform and nonreproducible distribution of intensity along the PS spill. These features tend to degrade the performance of the rf loops, thus inducing also changes in the position measurement on the first turn by the SPS BPM system, eventually leading to shot-by-shot measurement differences.

The adjustment of the horizontal trajectories of the five extracted turns from the PS requires special care due to the limited means available in the PS-SPS transfer line to perform turn-by-turn corrections of the beam trajectories. Indeed, more than a $5 \mathrm{~mm}$ difference in horizontal position at the injection point in the SPS was observed for the different PS-extracted turns.

In preparation for the resonant slow extraction at top energy, the fixed-target beams are operated with a working point below the third order resonance in the horizontal plane. The best transmission is achieved for vertical tunes close to the coupling resonance and above the half integer resonance. The nominal working point is therefore $Q_{x} / Q_{y}=26.62 / 26.58$. Good control of betatron tunes along the low energy part of the cycle and during transition crossing is crucial, and with the larger beams at injection, this is even more important for MTE beams.

\section{PERFORMANCE ANALYSIS FOR MTE BEAMS}

The quantitative analysis of the MTE technique is performed by considering the PS and SPS rings, first as separate entities, and then by looking for correlations between the two machines. This is imposed by the present state of MTE, in which fluctuations of the beam parameters are still present, although at a reduced level with respect to the initial beam commissioning. Afterwards, a direct comparison between CT and MTE is carried out to assess the improvements already achieved and the areas still requiring further attention.

For the performance analysis, certain time frames were chosen to analyze data from 2010 (for the initial MTE operational implementation) as well as from the 2015 run [25]. For 2015, one time frame was chosen as representative of the CT extraction technique on September 19th, which was near the end of the usage of CT. For the MTE data in 2015, the intensity $N_{p}$ was tested in three separate time frames to probe the following values: 1500,1800 , and 2000 , all in $10^{10} \mathrm{ppp}$. Note that while the first value had been used in operation for a few weeks, the intermediate
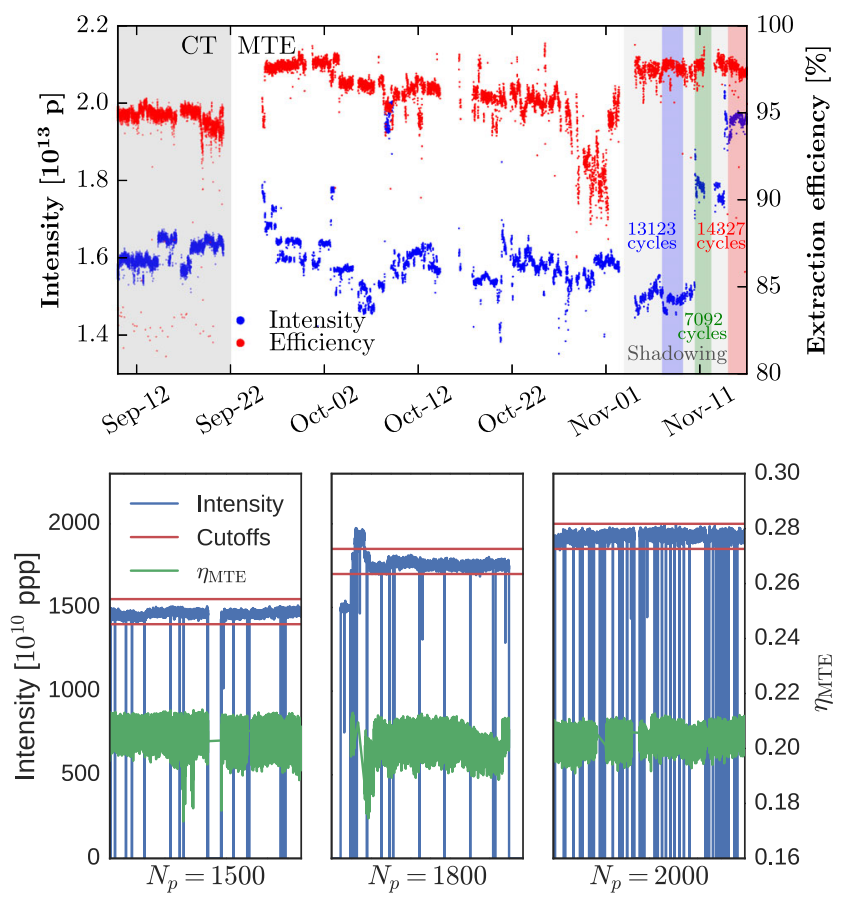

FIG. 10. Upper: Evolution of the PS beam intensity and extraction efficiency during the 2015 MTE run (from Ref. [25]). The three colored regions towards the end of the run correspond approximately to the selected time frames used in the analysis reported here. Bottom: PS intensity over time, shown together with the cuts in intensity, and $\eta_{\mathrm{MTE}}$.

one was used for SPS filling only for some hours, on the move toward $N_{p}=2000 \times 10^{10} \mathrm{ppp}$, which was the value kept until the end of the 2015 run. The overall picture is shown in Fig. 10 (upper part, from Ref. [25]), where the PS beam intensity and the extraction efficiency are plotted as a function of time.

To carry out the full performance analysis, certain cuts had to be made on the beam intensity entering the PS and SPS, so as to remove data that was anomalous and varied too widely from the configuration to which the machines were calibrated. In Fig. 10 (lower part), the raw data for the PS intensity is shown before any selection is made, along with the implemented cuts in intensity and corresponding $\eta_{\mathrm{MTE}}$ over time. The cuts used for all time frames are also summarized in Table IV. It is worth noting that the number of cycles with zero intensity, or in any case outside the range of the defined cuts, was correlated with the value of $N_{p}$. This is reflected in a larger fraction of rejected data, which, however, is completely uncorrelated with the transverse splitting, but rather based on the performance of the Linac 2 proton source.

As already mentioned in Sec. II A, although special beams have been prepared for MTE, the overall performance in terms of transfer losses between the PSB and the PS is essentially the same for CT and MTE. Therefore, the performance analysis presented in the following focuses on 
TABLE IV. Intensity selection ranges (all units, unless otherwise specified, are $10^{10} \mathrm{ppp}$ ). The large percentage of data removed from the sample corresponding to $N_{p}=1800$ is due to the period near the start in which the intensity was much too high.

\begin{tabular}{ccccc}
\hline \hline & Intensity & PS Acceptance & $\begin{array}{c}\text { SPS } \\
\text { Acceptance }\end{array}$ & $\begin{array}{c}\text { Removed } \\
\text { data [\%] }\end{array}$ \\
\hline \multirow{4}{*}{ MTE } & 1500 & $1400 \leq N_{p} \leq 1600$ & $2600 \leq N_{p}$ & 11.1 \\
& 1800 & $1700 \leq N_{p} \leq 1900$ & $3050 \leq N_{p}$ & 30.2 \\
CT & 2000 & $1850 \leq N_{p} \leq 2050$ & $3400 \leq N_{p}$ & 19.3 \\
\hline \hline
\end{tabular}

the PS and SPS rings. For a similar reason, the PS performance below $14 \mathrm{GeV} / c$ is not analyzed or discussed in the following given that no significant differences are found between CT and MTE.

\section{A. Proton synchrotron}

The analysis of the PS performance is based on three main figures-of-merit, namely $\eta_{\mathrm{MTE}}, \eta_{\mathrm{DC}}$, and $\eta_{\mathrm{Ext}}$. The first two have been already defined, while the last one is the extraction efficiency, obtained by comparing the intensity prior to extraction, as measured by a current transformer in the ring, with the measurement of a current transformer at the beginning of the transfer line. It is worth stressing that the accuracy with which the beam intensity is measured is not the same for the case of circulating or single pass beam, the latter being much less accurate.

To evaluate $\eta_{\mathrm{MTE}}$ and $\eta_{\mathrm{DC}}$, the extracted beam intensity $I(t)$ (also called spill in the following) must be examined. The spill can be acquired by either a high- or a lowsampling rate device. Given the large amount of data generated by the latter, the data used for the analysis reported in this paper is based on the low-sampling rate device. However, for a specific data sample a detailed comparison of $\eta_{\mathrm{MTE}}$ and $\eta_{\mathrm{DC}}$ provided by the two devices has been carried out and a very good correlation was found (featuring a correlation factor $\rho$ around 0.99).

An example of typical spills for both CT and MTE can be seen overlaid on top of each other in Fig. 11. These spills have been calculated by restoring the baseline linearity,

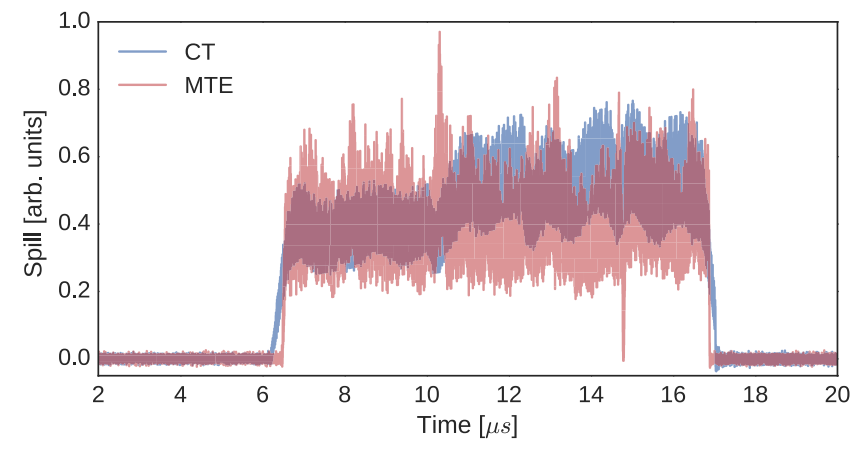

FIG. 11. Example spills for both CT and MTE. which is distorted by the electrical properties of the circuits, and by suppressing the resulting constant baseline [42]. It is important to note the two main differences between the two extraction techniques, namely the slope of the spill, both at the start and end of extraction, which is much steeper for MTE, due to faster kicker rise time in comparison with the CT fast dipoles, and the dip in intensity when the fifth turn is extracted, which is much lower for MTE, due to the direct losses on the magnetic septum.

Histograms of $\eta_{\mathrm{MTE}}$ and of $\eta_{\mathrm{DC}}$ can be seen in Fig. 12 (upper and lower part, respectively) and the distributions are close to the nominal values for each figure-of-merit.

A histogram of $\eta_{\text {Ext }}$ can be seen in Fig. 13 (upper part) and the independence of the distributions from beam intensity is clearly visible. Similarly, a transfer efficiency for the transfer line has been defined using as boundaries the first current transformer out of the PS ring, i.e., the same device used for $\eta_{\text {Ext }}$, and the last device before the injection point into the SPS. The distribution of this figure-of-merit is also shown in Fig. 13 (lower part). The distributions are sharply peaked, without large tails, thus indicating that the fluctuations in $\eta_{\mathrm{MTE}}$ are not affecting the performance of the beam transfer between the two rings (see later).

The last observation, already made in Ref. [25], is that only a very mild dependence on the beam intensity is found
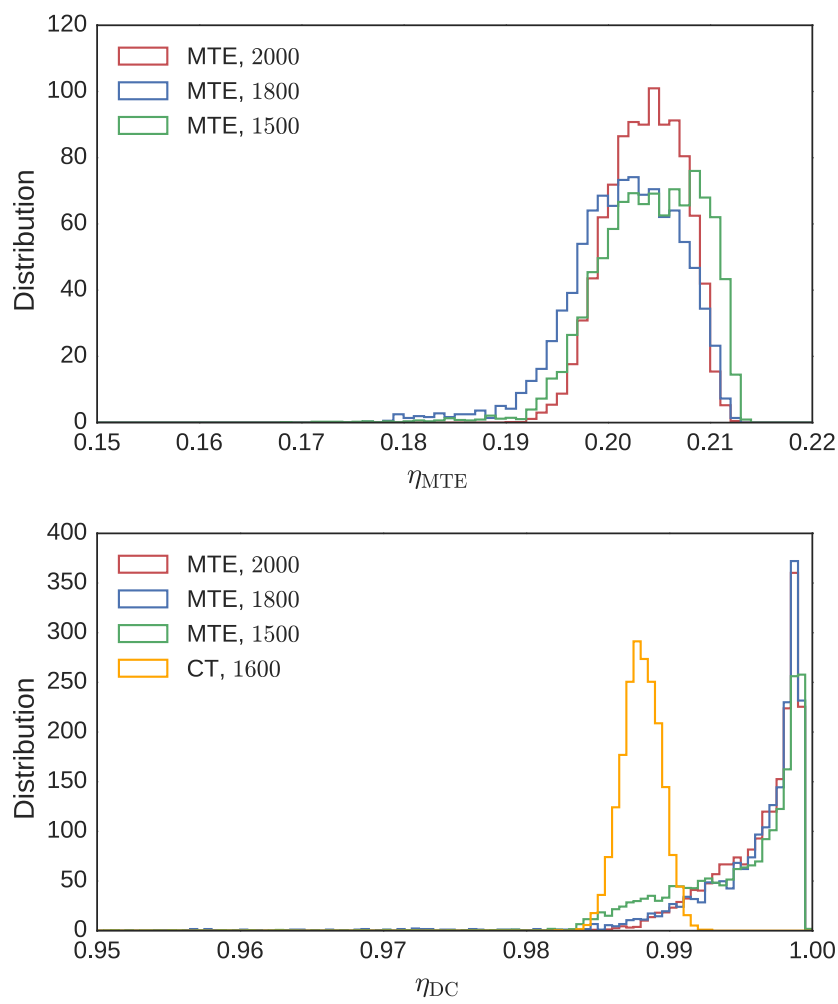

FIG. 12. Upper: Histogram showing the distribution of the MTE efficiency. Bottom: Histogram showing the distribution of the DC efficiency including CT data. The typical intensity values are in units of $10^{10} \mathrm{ppp}$ at extraction from the PS. 

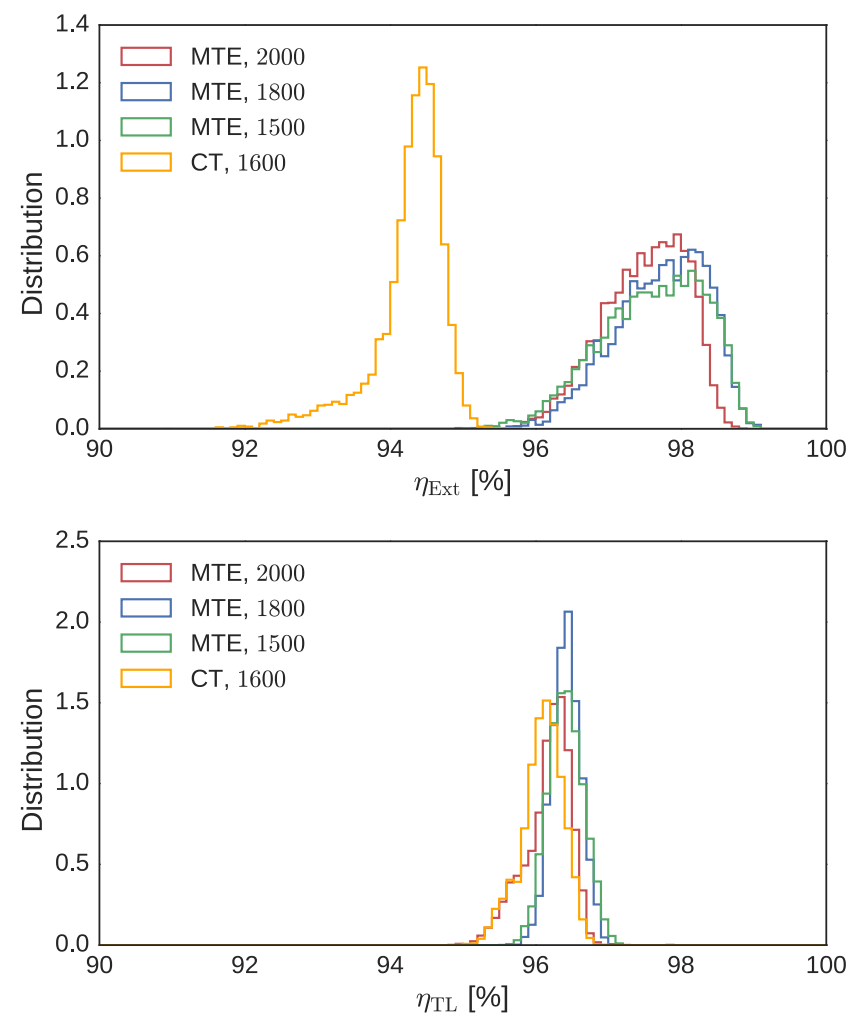

FIG. 13. Upper: Histogram showing the distribution of the PS extraction efficiency. Bottom: Histogram showing the distribution of the transfer line transmission efficiency. The typical intensity values are in units of $10^{10} \mathrm{ppp}$ at extraction from the PS.

in the 2015 data, which is certainly an important point for future developments with higher intensity beams.

\section{B. Super Proton Synchrotron}

While in the PS, the split beams are generated only prior to extraction, and the evaluation of the performance of the process is limited to the analysis of the final stages of the PS cycle. In the case of the SPS, it is important to consider the performance throughout the whole acceleration cycle, to assess whether there are critical stages for the MTE beam. The standard approach used to qualify the SPS performance is based on the definition of key times along the magnetic cycle at which the transmission efficiencies are examined. The names for these times (and their abbreviations) are as follows: (i) Injection (Inj), $1220 \mathrm{~ms}$ after the first injection (ii) Flat Bottom (FB), $1260 \mathrm{~ms}$ after the first injection (iii) Front Porch (FP), $1460 \mathrm{~ms}$ after the first injection (iv) Transition (Tr), $1530 \mathrm{~ms}$ after the first injection (v) Flat Top (FT), $4260 \mathrm{~ms}$ after the first injection. These times can also be seen in Fig. 14 where the evolution of the beam intensity and momentum is shown as a function of time. Note also that transmission efficiencies within the SPS are defined in terms of the transmission from the previous stage to the stage named. As an example, $\eta_{\operatorname{Tr}}$ is

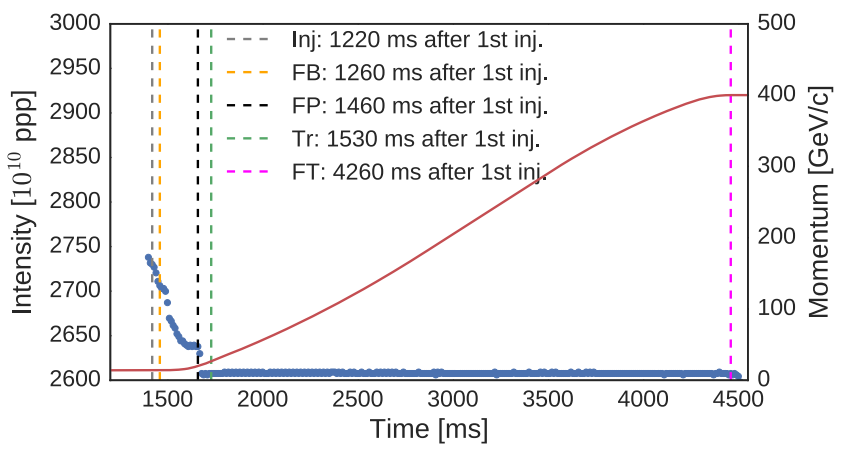

FIG. 14. Example of beam intensity in the SPS as a function of time.

defined as the percentage of intensity at FP that gets transmitted to $\mathrm{Tr}$.

It is worth stressing that two extractions from the PS are injected into the SPS, separated by $1.2 \mathrm{~s}$, the PS cycle length for accelerating protons to $14 \mathrm{GeV} / c$, which explains the flat bottom part of the SPS magnetic cycle. Therefore, the SPS injection efficiency is defined as being the sum of the circulating intensities, just after injection into the SPS, of each of the two beams, divided by the sum of the two beams' intensities in the transfer line just upstream of the injection point in the SPS.

The distributions of the efficiencies at the various key times for the three intensity values used in 2015 are reported in Fig. 15, including also comparative data from the CT. Wherever possible, the data from the 2010 MTE run is also included in the analysis. Similar to what was observed in the PS, a sizeable improvement is visible both in terms of mean and rms values of the distribution of the efficiency figures. Nevertheless, it is worth pointing out that $\eta_{\mathrm{FB}}$ for the MTE in 2010 is comparable with what was measured in 2015 for the lowest intensity used. This is possibly an indication of the reduction of SPS vertical aperture over time, as the vertical emittance of the MTE beam delivered by the PS is approximately constant over the years.

The other important aspect is that, unlike in the PS, where only mild dependence on beam intensity was observed, the SPS performance clearly depends on the beam intensity. In the flat bottom part specifically, and more generally in the low energy part of the SPS cycle, the intensity dependence can be explained in terms of the link between beam emittance and intensity, combined with the SPS aperture limitation in the vertical plane (see Sec. II C). This is also supported by the observation that the difference in transmission for the three beam intensities decreases with energy in the SPS, as the physical beam emittance shrinks, thus mitigating the aperture issue. The three MTE beam intensities do not lead to any measurable difference in performance beyond the transition energy and for this stage the beam losses are virtually zero. The nonmonotonic behavior of the beam losses vs. intensity is due to the fast 

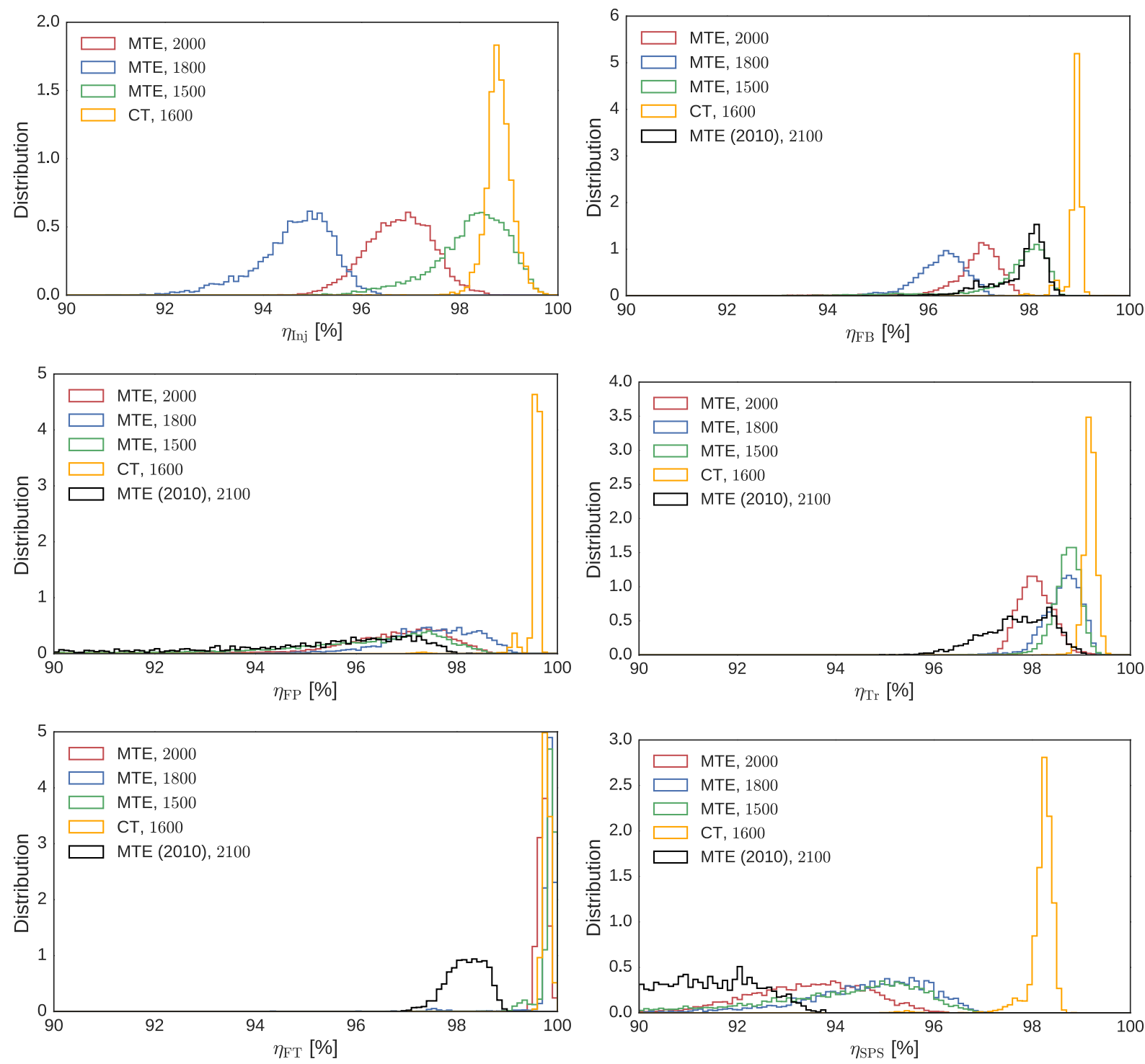

FIG. 15. Global picture of the distribution of the beam transmission through the SPS cycle at the key times used for the performance evaluation. The typical intensity values are in units of $10^{10} \mathrm{ppp}$ at extraction from the PS.

transition through the case $N_{p}=1800 \times 10^{10} \mathrm{ppp}$, which was not fully optimized like the other two intensity steps.

Another interesting point is the very much increased spread of the distributions of $\eta_{\mathrm{FP}}$ with respect to $\eta_{\mathrm{Inj}}$ and $\eta_{\mathrm{FB}}$. This could be linked with the combined effect of losses due to the limited vertical SPS acceptance (also affecting $\eta_{\text {Inj }}$ and $\left.\eta_{\mathrm{FB}}\right)$ and to longitudinal beam dynamics, like uncaptured beam that would be then lost at the beginning of acceleration. There are some hints that the MTE spill has more structure than the CT one (see the analysis carried out in Appendix C); however, until now it has not been possible to perform any measurement of the correlation between the spill structure and any of the efficiency figure-of-merit for the SPS.

A possible metric for the overall SPS performance is given by the SPS transmission efficiency $\eta_{\text {SPS }}$, defined in terms of the intensity at the end of the SPS cycle prior to beam extraction (at FT) divided by the sum of the two beams' intensities before ejection in the PS. The histogram is shown in Fig. 15 (lower right plot), where the overall improvement of the transmission efficiency over time is clearly visible and, as expected, features a dependence on beam intensity. $\eta_{\text {SPS }}$ is not the unique indicator of global performance for the MTE beam as one can also include the PS, providing a figure-of-merit for the efficiency between PS (before splitting) until SPS flat top. This indicator is called $\eta_{\text {Overall }}$ and its distribution is shown in Fig. 16. Once more, the MTE improvement over the years is clearly seen as well as a certain dependence on the beam intensity.

The last point to consider is that the previous efficiency indicators, which reflect a time dependence of the losses, 


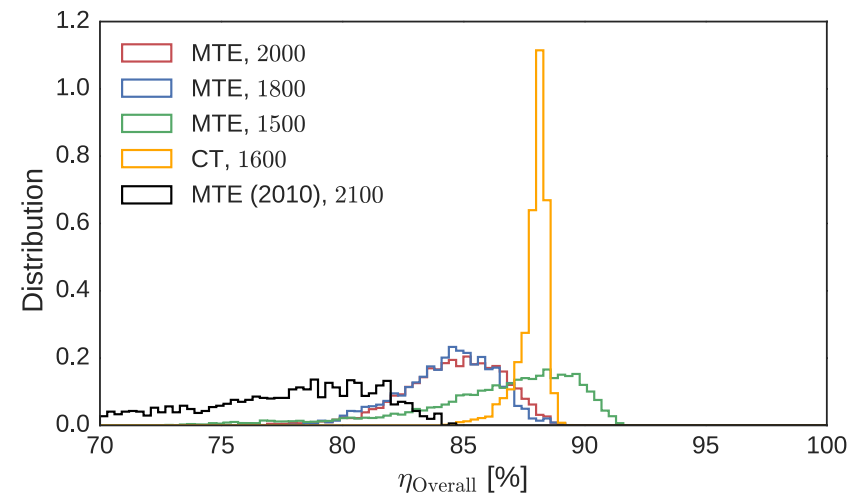

FIG. 16. Histogram showing the distribution of $\eta_{\text {Overall }}$. The typical intensity values are in units of $10^{10} \mathrm{ppp}$ at extraction from the PS.

can provide insight into the losses distributions as a function of beam momentum. This aspect is essential when evaluating the overall performance of MTE beams, i.e., looking at both machines, PS and SPS, and not focusing on a single one. Indeed, higher-energy particles produce different effects in terms of irradiation and activation, which makes the momentum distribution of beam losses an essential figure-of-merit. The situation is summarized in Fig. 17 where average beam intensity losses are shown as a function of beam momentum. After a full analysis of the intensity losses as a function of momentum for the SPS cycle, the most significant contribution to the lost intensity comes around $22 \mathrm{GeV} / \mathrm{c}$, corresponding to transition crossing, and the second significant contribution occurs at injection energy. In the other parts of the SPS cycle the losses are negligible. At injection (or close to injection) the losses are likely to be aperture-related, as previously discussed.

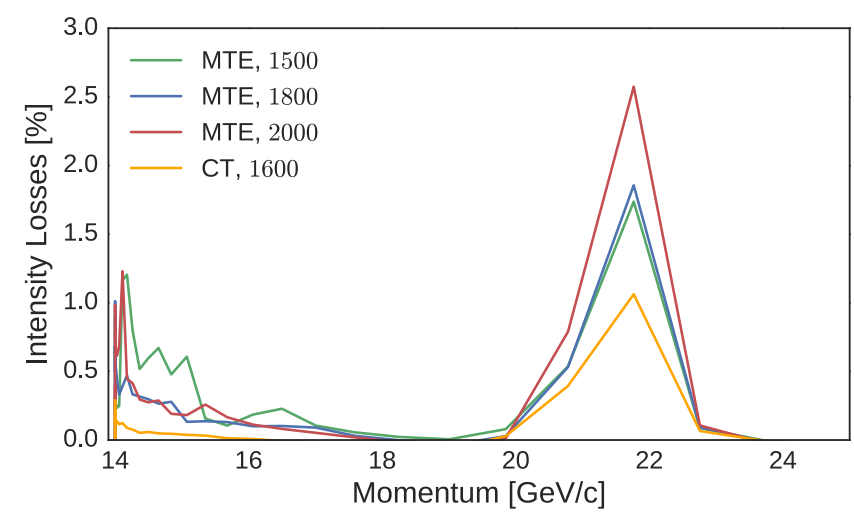

FIG. 17. Intensity losses in $\%$ as a function of momentum for 2015 data, both CT and MTE. The highest intensity losses (for all four time frames) come around $22 \mathrm{GeV} / \mathrm{c}$, corresponding to transition crossing, reaching as high as $2.5 \%$. The typical intensity values are in units of $10^{10} \mathrm{ppp}$ at extraction from the PS.

\section{Correlations between PS and SPS}

The presence of time variations in the main PS beam parameters like $\eta_{\mathrm{MTE}}$ also induces variations of the SPS performance, so that a statistical analysis of the correlation between the key efficiency indicators of the two machines is needed to assess which parameters rule the overall performance.

It is worth noting that whenever the PS and SPS data are to be considered globally, the two PS cycles, which are injected in the same SPS cycle, should in some sense be combined together. The analysis of the correlation of, e.g., $\eta_{\mathrm{MTE}}$ for two PS consecutive cycles shows a very strong correlation (higher than 0.80) for all data sets examined. This means that the time variations are on a longer time scale than that of the duration of the pairs of $14 \mathrm{GeV} / c$ PS cycles. For this reason, the efficiency indicators of PS cycles injected into the same SPS cycle have been averaged for usage in the analysis presented in the following. Furthermore, all SPS cycles consisting of only a single injection from the PS have been rejected and are not part of the data sample discussed.

The principle of the correlation analysis is based on the use of the PS and SPS efficiencies and the outcome is summarized in Fig. 18, where the elements of the correlation matrix are shown as heat maps. Given the intrinsic symmetries, only the lower-diagonal part of the matrix is shown here.

The first conclusion one can draw from the plots is that the overall pattern does not depend strongly on beam intensity. The second immediate conclusion from these heat maps is that there is a rather strong correlation between $\eta_{\mathrm{MTE}}$ and $\eta_{\mathrm{Ext}}$ for all three MTE time frames, which nevertheless represents a correlation between figures-ofmerit in the same ring. The third conclusion is that the correlation between $\eta_{\text {MTE }}$ and SPS efficiency figures is not that strong. This is an important outcome of the analysis, i.e., that the shape of the spill does not seem to be the main culprit for the SPS performance. In fact, $\eta_{\mathrm{MTE}}$ is positively correlated with losses earlier in the transmission through the SPS cycle (such as $\eta_{\mathrm{TL}}, \eta_{\mathrm{Inj}}$ ), but less correlated with later transmission efficiencies. Furthermore, the stronger correlation is only between $\eta_{\text {Ext }}$ and $\eta_{\text {Inj }}$, suggesting that improving the PS extraction will lead to more effective injection into the SPS. Note that $\eta_{\text {Inj }}$ is less strongly correlated with $\eta_{\mathrm{MTE}}$, thus indicating that a flat spill is less important, as far as SPS injection losses are concerned, than a clean PS extraction. It is also worth mentioning that the correlations are either close to zero or, in case they are larger, they are in the majority of cases positive, thus indicating that an improvement in the PS is also beneficial for the SPS.

To examine this further, the correlation plots for $\eta_{\mathrm{Ext}}$ and some of the SPS efficiencies against $\eta_{\mathrm{MTE}}$ are shown in Fig. 19. The progressive reduction of the correlation of SPS figures-of-merit and $\eta_{\text {MTE }}$ is clearly visible as well as a 


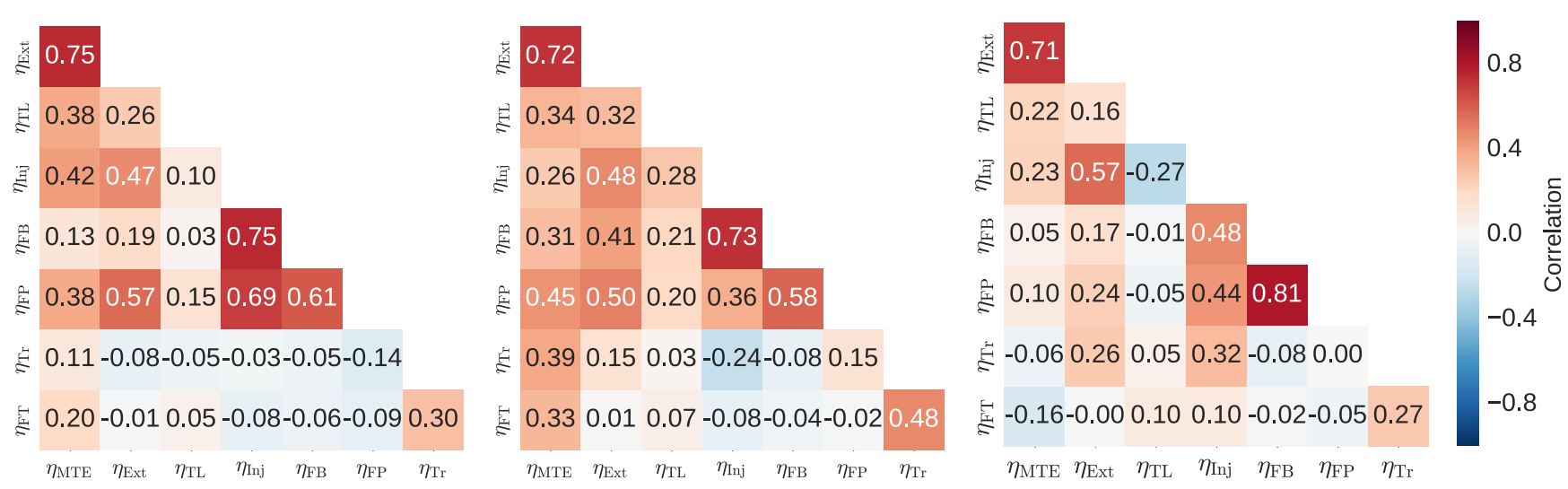

FIG. 18. Correlation heat maps for the figures-of-merit used in the analysis. The three plots refer to the different MTE beam intensities, namely $N_{p}=1500,1800,2000 \mathrm{ppp}$ for the left, center, and right panel, respectively. It is worth noting that the general tendency of correlations was found to be positive.

non-negligible number of outliers: in spite of their impact on the numerical value of the correlation, which could be higher in case of rejection of outliers, the plots clearly indicate that the reduction of correlation is real.

\section{Comparison between CT and MTE beams}

The last point that will be addressed is the direct comparison between CT and MTE in terms of beam performance as until now only the characterization of MTE has been discussed. The key quantities have been already shown in previous plots and in this section the main points will be presented. At the level of the PS ring, the MTE features a flatter spill than CT as can be seen in Fig. 12, where the distribution of $\eta_{\mathrm{DC}}$ is shown. In fact, even if MTE features longer tails in the distribution of $\eta_{\mathrm{DC}}$, the minimum value is still comparable with that of $\mathrm{CT}$ and the peak is much closer to 1. Moreover, MTE is also superior in terms of $\eta_{\text {Ext }}$ (see Fig. 13, upper), for which a clear shift of the position of the peak of the distribution is visible, according to the theoretical estimates [16]. On the other hand, the transfer line efficiency $\eta_{\mathrm{TL}}$ features very similar distributions for CT and MTE, in spite of the different optical configurations in the transfer line, such as the absence of emittance exchange for MTE. As far as the SPS is concerned, the situation is somewhat different. The overview of the figures-of-merit reported in Fig. 15 is rather clear: in general, CT performs better than MTE, with narrower peaks shifted toward higher values of the efficiencies than the MTE ones. The differences are particularly striking in the low-energy part of the SPS magnetic cycle, while they are less noticeable at higher energies and after transition no notable differences are found. Obviously, $\eta_{\mathrm{SPS}}$ is rather different (see Fig. 15, lower right) for CT and MTE, mainly due to the differences in the low-energy part of the SPS cycle. A somewhat different situation is found when considering $\eta_{\text {Overall }}$, where the main difference between CT and MTE is the spread of the distributions, while the mean values are very comparable.
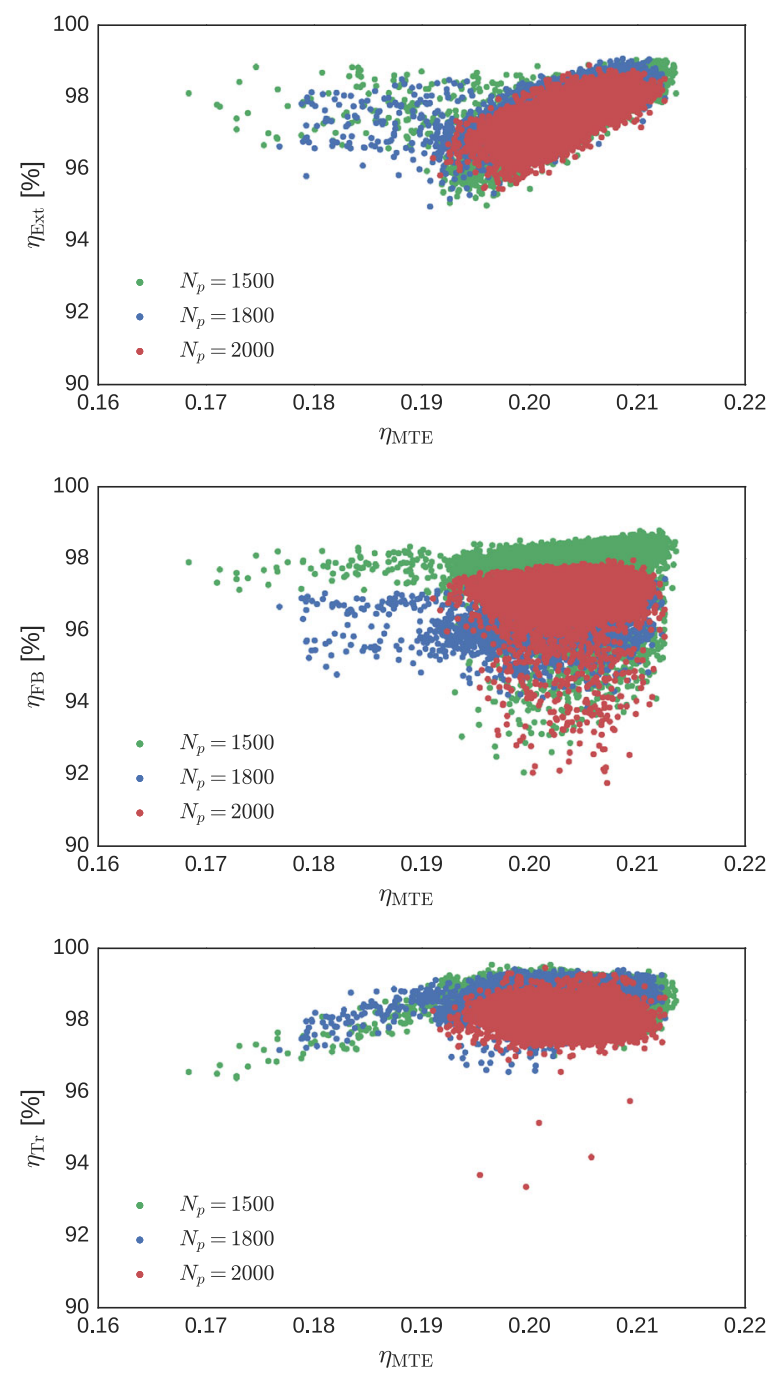

FIG. 19. Example of correlation plots between PS and SPS efficiency figures of merit. The progressively lower correlation between $\eta_{\text {MTE }}$ and transmission efficiencies for later stages in the SPS cycle is clearly visible. The typical intensity values are in units of $10^{10} \mathrm{ppp}$ at extraction from the PS. 

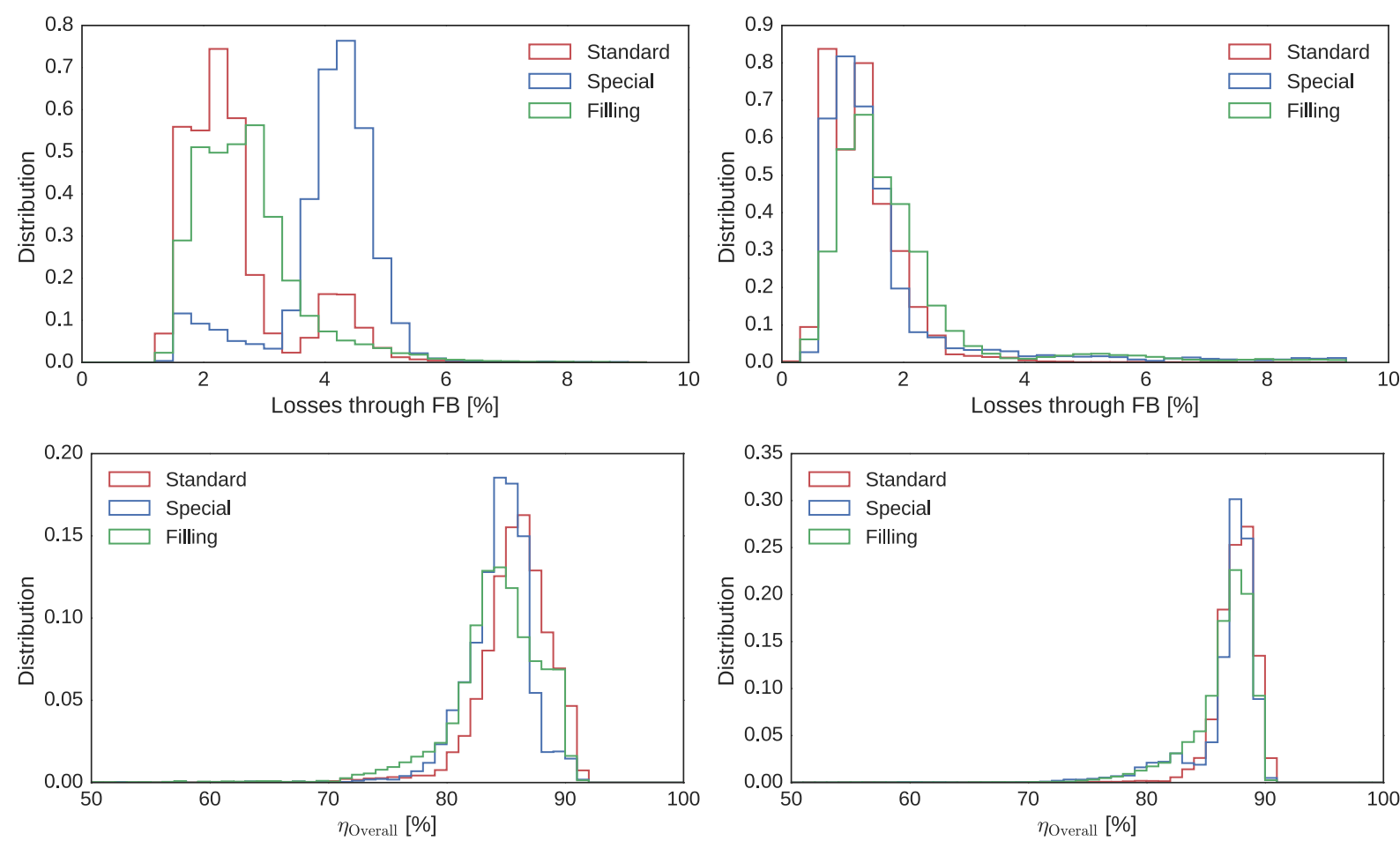

FIG. 20. Transmission efficiency $\eta_{\mathrm{FB}}$ (upper row) and $\eta_{\text {Overall }}$ (bottom row) for MTE (left column) and CT (right column) for the various types of SPS super-cycles (Standard for the normal super-cycle; Special for super-cycle for beam physics development studies; Filling for LHC filling super-cycle). The difference is clear and mainly stems from the different efficiency in the low-energy part of the SPS cycle.

The losses vs. beam momentum shown in Fig. 17 confirms what was already mentioned, i.e., in the SPS for the same beam intensity the losses for MTE are larger than for CT up to around transition and then the differences are no longer relevant.

Another specific aspect that must be addressed using the SPS efficiencies defined earlier is the sensitivity of MTE or CT beams to the composition of the SPS super-cycle. In fact, a dynamic super-cycle composition can be defined so that several beams can be produced, like cycles for the filling of the LHC ring, or to perform special studies in view of future developments. The change of super-cycle can generate differences in the magnetic hysteresis and remnant fields at injection, which could have an impact on the performance of MTE or CT production processes. In Fig. 20 the main results of this analysis have been summarized. The difference in $\eta_{\mathrm{FB}}$ for MTE and CT is clearly visible and it is the main source of difference in $\eta_{\text {Overall }}$. At low energy the mean value of the distribution of $\eta_{\mathrm{FB}}$ depends strongly on the SPS super-cycle for MTE, while the average is approximately constant for CT. This is possibly linked with the aperture issues affecting MTE beams in the vertical plane. In fact, any magnetic perturbation of the SPS configuration might turn into a variation of orbit, which, in case of a beam emittance close to the ring acceptance, might turn into increased beam losses and hence reduced efficiency.
In terms of correlation between PS and SPS performance, it is interesting to compare the heat maps for MTE, as reported in Fig. 18, and the corresponding situation for $\mathrm{CT}$, as reported in Fig. 21. The difference between MTE and $\mathrm{CT}$ is remarkable: indeed, while some correlation can be found for the MTE case, for CT all correlations are weak, the largest ones being of the order of 0.5 in absolute value. The negative correlation between $\eta_{\mathrm{TL}}$ and $\eta_{\mathrm{Inj}}$ and the positive correlation between $\eta_{\mathrm{FB}}$ and $\eta_{\mathrm{FP}}$ should both be noted. This is yet another confirmation that the spill profile,

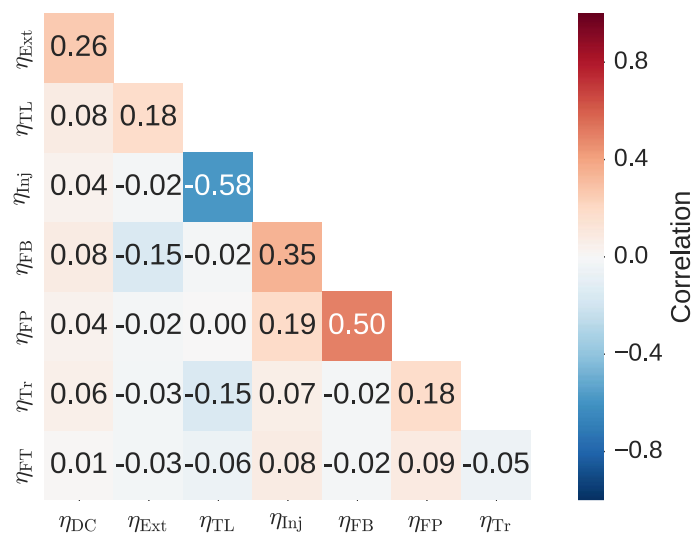

FIG. 21. Correlation heat map for the figures of merit used in the analysis of CT performance. 
as described by $\eta_{\mathrm{MTE}}$ or $\eta_{\mathrm{DC}}$, is essentially uncorrelated to the SPS performance. Finally, it is worth stressing that this observation cannot be used to draw a conclusion on the lack of correlation between the various figures of merit for the CT case, as the weak correlation could be (all or in part) due to the smaller fluctuations in the efficiency figures-of-merit for CT than for MTE (see, e.g., Fig. 15).

As a last point, another way of directly comparing CT and MTE consists of examining the information from the individual beam loss monitors (BLMs) that are installed along both the PS and SPS circumference. For the PS BLMs, Fig. 22 (upper part) shows a striking improvement of MTE with respect to CT. This is, of course, the consequence of the MTE principle, which avoids the beam slicing with an electrostatic septum, leading to a dramatic reduction of beam losses around the PS ring. Note that the plot has been generated using data with $N_{p}=1500$ (the closest intensity to that of the CT time frame) and the overall pattern of losses does not differ when other intensities are used. Furthermore, the readings, when normalized by the average value of the beam intensity, are approximately constant. The SPS situation is also visible in Fig. 22 (lower part). These losses are scaled by the intensity of the beam, so as to give a direct comparison. In most of the BLMs, there are similar levels of losses, but in BLM 121 there is a clear intensity
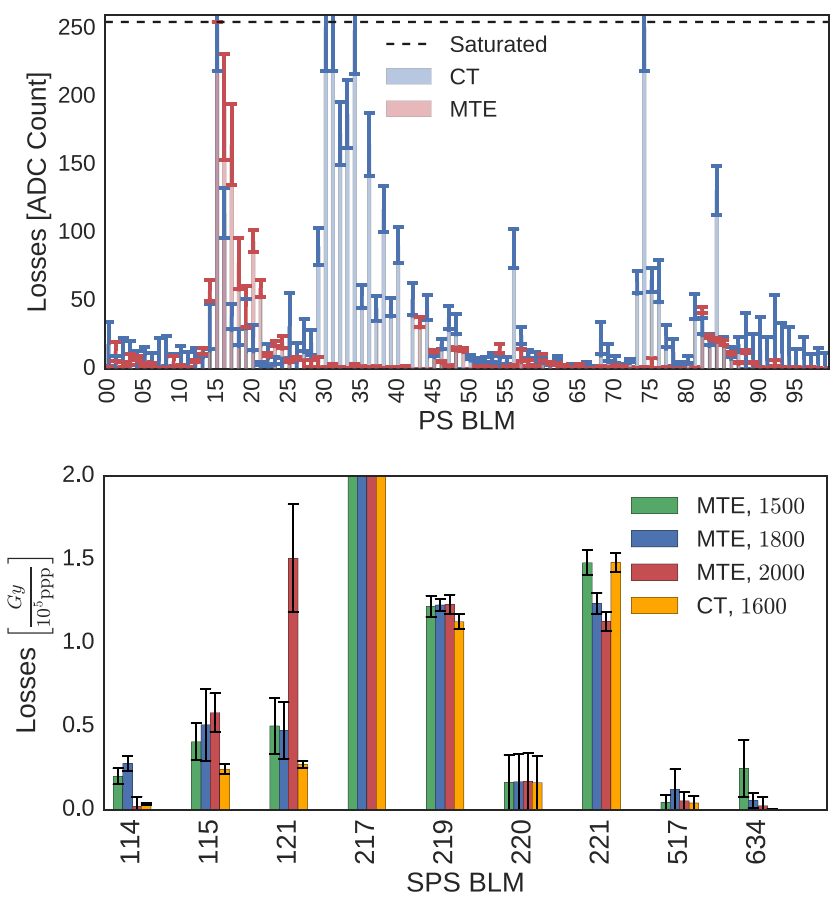

FIG. 22. BLM readings for MTE and CT in the PS (upper) and SPS (lower) rings. The bars represent the average, while the error bars the rms values of the BLMs. For the PS case the readings are given in ADC counts, while for the SPS, the readings have been normalized to the beam intensity. The typical intensity values are in units of $10^{10} \mathrm{ppp}$ at extraction from the PS.

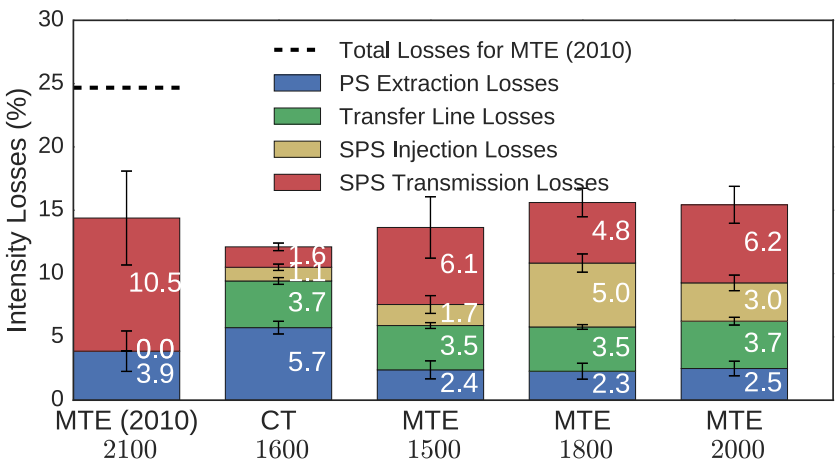

FIG. 23. Bar plot of losses for MTE and CT beams. The height of each bar represents the total losses, from before ejection in the PS, to the start of Flat Top in the SPS. The typical intensity values are in units of $10^{10} \mathrm{ppp}$ at extraction from the PS.

dependence, with an increase of a factor of 2 or more when the highest intensity $\left(N_{p}=2000\right)$ is used. The analysis of the signal of the BLM 121 revealed that the beam losses occur at the moment of the second injection and are generated by the beam uncaptured after the first injection. This fraction of beam is deflected by the injection kicker, which is located $90^{\circ}$ upstream of the BLM location, and is lost. Therefore, the increase of losses at this location is a a sign of increased level of uncaptured beam as a function of intensity.

To summarize the current breakdown of where the losses come from in the PS to SPS extraction mechanism, Fig. 23 shows the losses in each of the four major sections (PS Extraction, Transfer Lines, SPS Injection, and SPS overall transmission) stacked so as to view the overall beam losses. For the 2010 run, no data is available to evaluate the losses in the transfer line and at SPS injection, so they have been left as $0.0 \%$ (to only compare like to like), but the dashed line at the top of the figure shows the overall losses in 2010. The first point to note is the huge improvement for the global MTE performance since 2010. The improvement in the PS losses thanks to MTE should be stressed, while the situation in the transfer line is very much the same for CT and MTE. The situation in the SPS favors CT, as far as beam losses are concerned, even if globally, the combined losses between PS and SPS are almost the same for the two beam types. A last comment is particularly relevant: it should not be forgotten that CT was implemented in the 1970s. Hence, it was fully optimized over the course of several decades, and the level of losses shown in Fig. 23 are the results of a long and meticulous work. Clearly, MTE is comparatively very new and still has room for improvements.

\section{CONCLUSIONS AND OUTLOOK}

In this paper the first performance of the CERN injector complex with transversely split beams has been reviewed 
and presented in detail. The data are those from the first operational MTE run in 2015.

The PS performance with MTE beams has been greatly improved with respect to the first short run in 2010. The main goals of increasing the extraction efficiency and of reducing the PS ring irradiation have been fully achieved, with MTE surpassing the CT performance in agreement with expectations. Moreover, the dummy septum has been successfully commissioned and put in operation providing the required shadowing of the magnetic septum, without interfering with the extraction of the non-MTE beams.

For the first time, a detailed analysis of the SPS performance could be carried out, including also correlation between the two main rings, i.e., PS and SPS. The overall efficiency is very similar between MTE and CT, the first being superior in the PS and the latter in the SPS. The largest difference in terms of SPS performance is observed in the low-energy part of the cycle: the larger vertical emittance of the MTE beam generates beam losses due to a reduction of the vertical acceptance of the SPS. This is not felt as an intrinsic limitation of the MTE beam, as it could be overcome by a global realignment of the SPS ring so to restore a similar acceptance to what was available in the past. The possibility that a fraction of the losses in the early part of the SPS cycle are due to uncaptured beam will be explored by trying to establish a correlation between these losses and the high-frequency structure on the spill (see Appendix C). Another point to note when comparing CT and MTE beams is that the former has been optimized over many years, while the latter has been in operation for only a few months. All of this indicates that there is still room for improvement in the MTE performance, mainly in the SPS.

Efforts are continually devoted to better understanding of the origin of the fluctuations of $\eta_{\mathrm{MTE}}$ in the PS and a vigorous effort to reduce the ripple of specific power converters is ongoing and the beneficial impact is expected to be seen during the 2017 physics run.

The 2016 fixed target physics run at the SPS has started using MTE as operational beam. This entailed developing a lower intensity MTE, as for early commissioning after shutdowns and longer technical stops, the SPS requires a lower intensity batch $\left(\approx 4 \times 10^{12} \mathrm{ppp}\right)$ ideally of the length of the nominal batch of $10.5 \mu \mathrm{s}$. Reducing the MTE beam intensity in the PS requires readjusting the parameters for the creation of the islands and is hence not a completely transparent operation. This new type of MTE beam was successfully delivered to the SPS, and currently the fixed target experiments are using MTE beams at typical beam intensities of $1000 \times 10^{10} \mathrm{ppp}$ in view of higher intensities for the 2017 run. Under these conditions, CT remains only a backup option in case of major issues with MTE.

\section{ACKNOWLEDGMENTS}

We would like to acknowledge the CERN PS-Booster, PS, and SPS Operations crew for constant help during the measurement sessions throughout the years of MTE commissioning. Our warm thanks go to G. Arduini and J.-P. Burnet for the constant support and encouragement provided during the long and intense commissioning period. It is a pleasure to mention with warm appreciation the fruitful collaboration with A. Bazzani, A. Franchi, and G. Turchetti. With them we shared the pleasure of discussing several theoretical aspects related with resonance crossing in view of making a practical application out of these concepts. We also shared some good times in devising new beam manipulations based on nonlinear beam dynamics and stable resonances. The results obtained by the end of 2015 represent the final achievement of intense efforts that started back in 2002. Throughout the years several people contributed to these studies and to the struggle to turn the original idea into an operational technique. It is more than appropriate to give credit to three persons who played a key role in this enterprise. They provided the initial momentum and the strong motivation that kept the activities in motion until the successful end, namely R. Cappi, who is indeed one of the originators of the proposal to replace CT with an extraction mode based on transverse beam splitting, K. Hübner, and J.-P. Riunaud.

\section{APPENDIX A: PROPERTIES OF THE FIGURES-OF-MERIT $\eta_{\text {MTE }}$ AND $\eta_{\text {DC }}$}

Using the notation of Sec. II B and assuming that the PS spill $I(t)$ is a step-wise constant function of the form

$I(t)=\langle I\rangle+\Delta_{j} \quad$ for $(j-1) \tau<t \leq j \tau \quad$ and $\quad 1 \leq j \leq 5$

where

$$
\langle I\rangle=\frac{1}{T} \int_{0}^{T} I(t) d t \quad \text { and } \quad \sum_{j=1}^{5} \Delta_{j}=0,
$$

and $T=5 \tau$ then Eq. (3) reads:

$$
\eta_{\mathrm{DC}}=\frac{1}{1+\frac{1}{5\langle I\rangle^{2}} \sum_{j=1}^{5} \Delta_{j}^{2}} .
$$

Equation (A3) is suitable for the analysis of the performance of beams extracted like the CT.

Note that the maximum of $\eta_{\mathrm{DC}}$ is achieved when $\Delta_{j}=0$ for $1 \leq j \leq 5$.

Equation (A1) allows rewriting $\eta_{\mathrm{MTE}}$ as

$$
\eta_{\mathrm{MTE}}=\frac{1}{5}\left[1+\frac{1}{4\langle I\rangle} \sum_{j=1}^{4} \Delta_{j}\right]
$$

or, alternatively 


$$
\eta_{\mathrm{MTE}}=\frac{1}{5}\left[1-\frac{\Delta_{5}}{4\langle I\rangle}\right]
$$

Note that if $\eta_{\mathrm{MTE}}=1 / 5$, corresponding to equal sharing of intensity over the five extracted turns, then $\Delta_{j}=0$ for $1 \leq j \leq 5$ and $\eta_{\mathrm{DC}}=1$.

By using the previous expressions for $\eta_{\mathrm{MTE}}$, it is possible to recast Eq. (A3) in a form which shows the dependence on $\eta_{\mathrm{MTE}}$, namely

$$
\eta_{\mathrm{DC}}=\frac{1}{1+\frac{32}{5}\left(5 \eta_{\mathrm{MTE}}-1\right)^{2}-\frac{1}{5\langle I\rangle^{2}} \sum_{j, k=1}^{4}{ }^{\prime} \Delta_{j} \Delta_{k}}
$$

where the symbol $\sum_{j, k}^{\prime}$ indicates that the sum is performed only for $j \neq k$.

The rather general assumption (A1) can be specialized to the case of MTE beams. In this case one has that all $\Delta_{j}$ for $j \leq 4$ are equal, so that only two free parameters describe the extracted beam intensity, namely $\Delta$ (representing the common value of $\Delta_{j}$ for $\left.j \leq 4\right)$ and $\Delta_{5}$. In this case it is immediate to see that

$$
\eta_{\mathrm{DC}}=\frac{1}{5} \frac{1}{1-8 \eta_{\mathrm{MTE}}+20 \eta_{\mathrm{MTE}}^{2}} .
$$

\section{APPENDIX B: HARMONIC ANALYSIS OF INTENSITY SPILL FOR MTE AND CT BEAMS}

Harmonic analysis of the extracted spill can provide additional insight into its features. Assuming the form (A1) for $I(t)$, then the amplitude of the Fourier component of order $n$ is given by

$$
\begin{aligned}
c_{n} & =\frac{1}{T} \int_{0}^{T} I(t) e^{-\frac{2 \pi i n}{T}\left(t+\frac{T}{2}\right)} d t \\
& =\frac{e^{-\pi i n}}{T} \sum_{j=1}^{5} \int_{(j-1) T / 5}^{j T / 5}\left[\langle I\rangle+\Delta_{j}\right] e^{-\frac{2 \pi i n}{T}\left(t+\frac{T}{2}\right)} d t \\
& =\frac{(-1)^{n+1}}{2 \pi i n}\left(1-e^{\frac{2 \pi i n}{5}}\right) \sum_{j=1}^{5} \Delta_{j} e^{-\frac{2 \pi i n}{5} j} .
\end{aligned}
$$

This means that harmonics of the fundamental frequency $1 / T=1 / 10.5 \mathrm{MHz} \approx 95 \mathrm{kHz}$ for the PS case are present in the spectrum, with the corresponding amplitude $\left|c_{n}\right|$ decreasing as $1 / n$. It is worth stressing that the components $c_{5 k}$ are naturally suppressed from the spectrum.

In the general case, the expression (B1) cannot be expressed only in terms of, e.g., $\eta_{\mathrm{DC}}$ or $\eta_{\mathrm{MTE}}$, but an alternative expression of $\left|c_{n}\right|$ can be given highlighting its dependence on $\eta_{\mathrm{DC}}$, namely

$$
\begin{aligned}
\left|c_{n}\right|= & \frac{1}{(2 \pi n)^{2}}\left|1-e^{\frac{2 \pi i n}{5}}\right| 5\langle I\rangle^{2} \\
& \times\left[\frac{1-\eta_{\mathrm{DC}}}{\eta_{\mathrm{DC}}}+\frac{1}{5\langle I\rangle^{2}} \sum_{j, k=1}^{5} \Delta_{j} \Delta_{k} e^{-\frac{2 \pi i n}{5}(j-k)}\right] .
\end{aligned}
$$

For the MTE case, however, the previous results can be cast in a simpler form. Indeed, by using the fact that the spill can be described by two free parameters, only ( $\Delta$ and $\Delta_{5}$ ), one has

$$
c_{n}={\frac{(-1)^{n+1}}{2 \pi i n}}^{\Delta_{5}} \frac{5 \eta_{\mathrm{MTE}}-1}{1-4 \eta_{\mathrm{MTE}}}\left(1-e^{\frac{2 \pi i n}{5}}\right)
$$

where the following relation has been used

$$
\eta_{\mathrm{MTE}}=\frac{\Delta}{4 \Delta+\Delta_{5}} .
$$

Of course, whenever all $\Delta_{j}$ are equal, or for the MTE case $\Delta=\Delta_{5}$ corresponding to $\eta_{\mathrm{MTE}}=0.2$, then $c_{n}=0 \forall n>0$ while $c_{0}=\langle I\rangle$. In all other cases a direct relationship between $c_{n}$ and $\eta_{\mathrm{MTE}}$ is present.

The previous models can be made more realistic by assuming that the constant steps $\Delta_{j}$ are indeed modulated. In this case, the assumptions Eqs. (A1) and (A2) are still valid, but now

$$
\Delta_{j}(t)=\left\langle\Delta_{j}\right\rangle+f_{j}(t) \quad 1 \leq j \leq 5
$$

where $f_{j}(t)$ has zero mean. Equation (B1) can be recast in the following form

$$
\begin{aligned}
c_{n}= & \frac{(-1)^{n+1}}{2 \pi i n}\left(1-e^{\frac{2 \pi i n}{5}}\right) \sum_{j=1}^{5}\left\langle\Delta_{j}\right\rangle^{-\frac{2 \pi i n}{5} j} \\
& +\frac{1}{T} \sum_{j=1}^{5} \int_{(j-1) T / 5}^{j T / 5} f_{j}(t) e^{-\frac{2 \pi i n}{T}\left(t+\frac{T}{2}\right)} d t .
\end{aligned}
$$

It is readily seen that in this case $c_{5 k}$ is not zero anymore given the additional term depending of $f_{j}(t)$. The value of $\left|c_{n}\right|$ can be found easily from Eq. (B6), and it comes out that it depends on $\eta_{\mathrm{DC}}$ with a term of the form (B2) and on two additional terms: one depending on $f_{j}(t)$, only, and a mixed term including both $f_{j}(t)$ and $\Delta_{j}$. Of course, the same occurs for the form (B3).

\section{APPENDIX C: HARMONIC ANALYSIS APPLIED TO MEASURED INTENSITY SPILLS FOR MTE AND CT BEAMS}

While the low-resolution data of the spill have been used to extract information on the efficiencies of the MTE process (see Sec. III A), the high-resolution data are ideal to perform the harmonic analysis detailed in the previous 
sections. A fast Fourier transform (FFT) can be computed to investigate the frequencies of signals that make up the spill, which is shown in Fig. 24.

The high-amplitude component at $200 \mathrm{MHz}$ is by design: this is the frequency by which the spill is modulated in the PS. High-order harmonics are also present, while any other frequencies come from some other sources. For example, the strong signal near $192.5 \mathrm{MHz}$ is due to the $h=16$ bunching. In fact, the beam at flat top consists of 16 bunches and then it is debunched prior to extraction (see Fig. 3). However, the relatively short time allocated for this beam manipulation implies that a residual modulation of the beam intensity at $h=16$ is present. This is indeed the case, as can be seen in the center and lower plot of Fig. 24.

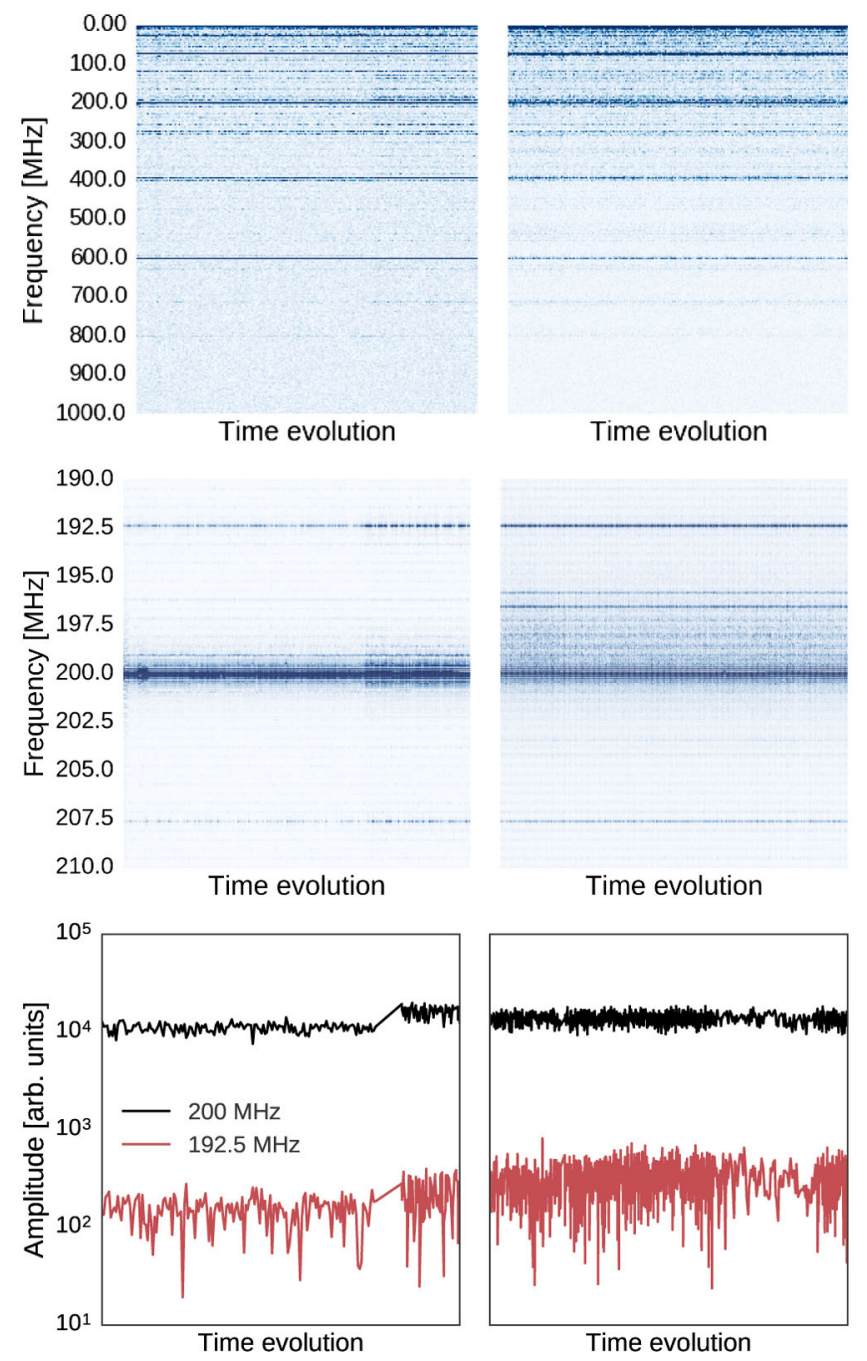

FIG. 24. Amplitudes of frequencies from FFT for CT (left column) and MTE (right column) extracted spills as a function of time. The upper plot shows all frequencies present in the spectrum, while in the center plot a zoom in the region around the $200 \mathrm{MHz}$ component is shown. The bottom plot shows the evolution over time of the $200 \mathrm{MHz}$ and of the $192.5 \mathrm{MHz}$ components only.
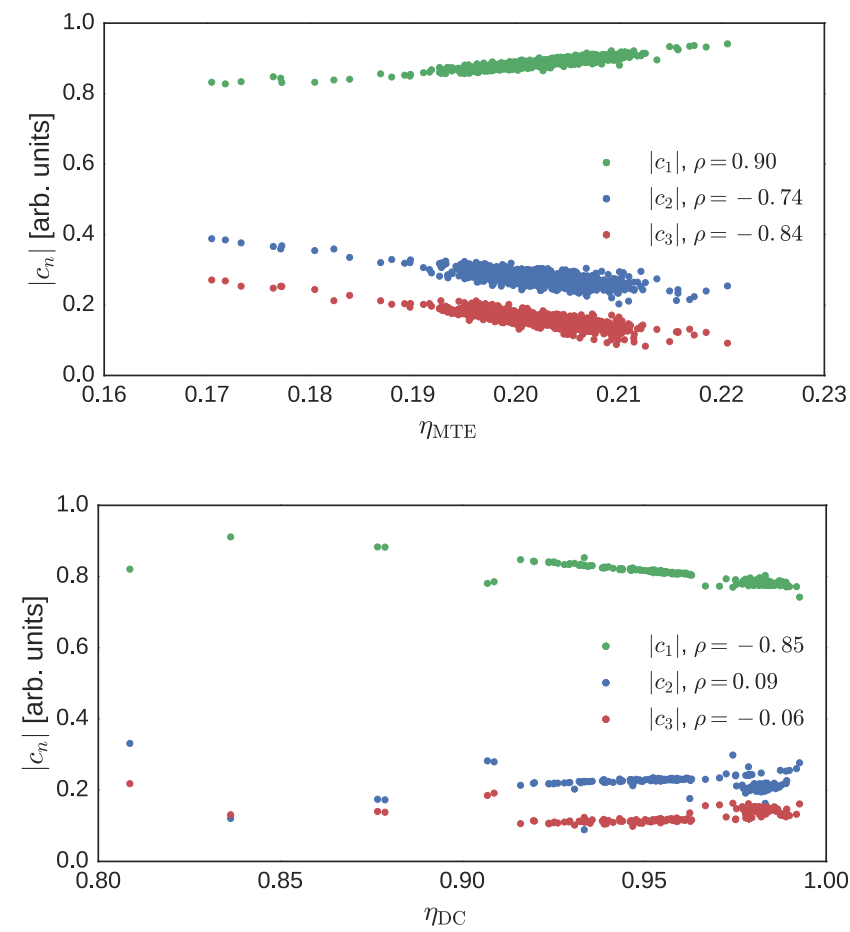

FIG. 25. Investigating relationships between $\left|c_{n}\right|$ and $\eta_{\mathrm{MTE}}, \eta_{\mathrm{DC}}$.

A comparative analysis between the CT and MTE case shows that, globally, they are very similar, even if the lowfrequency components, i.e., lower than $100 \mathrm{MHz}$ are more important for MTE than for CT (Fig. 24, upper plot). Concerning the spectrum content around the main $200 \mathrm{MHz}$ frequency, the component at $192.5 \mathrm{MHz}$ is present in both cases, although, with a stronger amplitude variation for MTE. Indeed, even the $200 \mathrm{MHz}$ component features some amplitude variations over time that are more important for MTE. Note that the data used for the analysis presented here were not taken during periods when the beam was delivered to the SPS. Therefore, it is not possible to assess whether there is any correlation with the spectrum structure and the performance of the SPS ring, in particular the losses during acceleration and close to transition energy. This aspect will be considered in future studies.

Another aspect analyzed using the experimental data is the dependence of some key frequency components on the typical efficiency parameters like $\eta_{\mathrm{MTE}}$ and $\eta_{\mathrm{DC}}$. The results of the numerical analysis are shown in Fig. 25 in which the dependence of $\left|c_{n}\right|, 1 \leq n \leq 3$ (according to the notation used in Appendix B) on $\eta_{\mathrm{MTE}}$ (upper) and $\eta_{\mathrm{DC}}$ (lower) is shown.

A good correlation is clearly visible, confirming the theoretical analysis carried out before. However, it is clear that $\left|c_{n}\right|$ does not go to zero for $\eta_{\mathrm{MTE}}=0.2$. This is the sign that an additional modulation of the spill is present, beyond the structure generated by a nonuniform sharing of beam intensity between islands and core, according to what is predicted by Eq. (B6). Note also that the sampling rate is 
not high enough to resolve the main frequency of $\approx 95 \mathrm{kHz}$ and its harmonics. In the future, correlation between the amplitudes of these special harmonic components and the SPS performance might be investigated.

[1] C. Bovet, D. Fiander, L. Henny, A. Krusche, and G. Plass, in Particle Accelerator Conference, San Francisco, CA, USA, 1973, edited by D. W. Dupen (IEEE, New York, 1973), p. 438.

[2] D. C. Fiander, D. Grier, K.-D. Metzmacher, and P. Pearce, A modulated fast bump for the CPS continuous transfer, IEEE Trans. Nucl. Sci. 24, 1340 (1977).

[3] K. Elsener et al., Report No. CERN-98-02, 1998.

[4] J. Barranco García and S. Gilardoni, Simulation and optimization of beam losses during continuous transfer extraction at the CERN Proton Synchrotron, Phys. Rev. ST Accel. Beams 14, 030101 (2011).

[5] R. Cappi and M. Giovannozzi, Report No. CERN PS 2002083 (AE), 2002.

[6] R. Cappi and M. Giovannozzi, Multiturn extraction: performance analysis of old and new approaches, Nucl. Instrum. Methods Phys. Res., Sect. A 519, 442 (2004).

[7] R. Cappi and M. Giovannozzi, Novel Method for Multiturn Extraction: Trapping Charged Particles in Islands of Phase Space, Phys. Rev. Lett. 88, 104801 (2002).

[8] R. Cappi, M. Giovannozzi, M. Martini, E. Métral, G. Métral, R. Steerenberg, and A.-S. Müller, in Proceedings of the 2003 Particle Accelerator Conference, Portland, OR (IEEE, New York, 2003), p. 388.

[9] R. Cappi, S. Gilardoni, M. Giovannozzi, M. Martini, E. Métral, A.-S. Müller, A. Sakumi, and R. Steerenberg, in Proceedings of the 9th European Particle Accelerator Conference, Lucerne, 2004 (EPS-AG, Lucerne, 2004), p. 175.

[10] M. Giovannozzi, R. Cappi, S. Gilardoni, M. Martini, E. Métral, R. Steerenberg, and A.-S. Müller, in Proceedings of the 21st Particle Accelerator Conference, Knoxville, TN, 2005 (IEEE, Piscataway, NJ, 2005), p. 117.

[11] S. Gilardoni, M. Giovannozzi, M. Martini, E. Métral, P. Scaramuzzi, R. Steerenberg, and A.-S. Müller, Resonant multi-turn extraction: Principle and experiments, Nucl. Instrum. Methods Phys. Res., Sect. A 561, 249 (2006).

[12] S. Gilardoni, M. Giovannozzi, M. Martini, E. Métral, P. Scaramuzzi, R. Steerenberg, and A.-S. Müller, Experimental evidence of adiabatic splitting of charged particle beams using stable islands of transverse phase space, Phys. Rev. ST Accel. Beams 9, 104001 (2006).

[13] A. Franchi, S. Gilardoni, and M. Giovannozzi, Progresses in the studies of adiabatic splitting of charged particle beams by crossing nonlinear resonances, Phys. Rev. ST Accel. Beams 12, 014001 (2009).

[14] O. E. Berrig, J. Borburgh, J.-P. Burnet, R. Cappi, M. Giovannozzi, W. Kalbreier, M. Martini, A.-S. Müller, E. Métral, K.-D. Metzmacher, J.-P. Riunaud, A. Sakumi, P. Scaramuzzi, L. Sermeus, R. Steerenberg, and T. Zickler, Report No. CERN-AB-2004-003-ABP, 2004.
[15] M. Giovannozzi (PS Multi-Turn Study Group), in Proceedings of the 10th European Particle Accelerator Conference, Edinburgh, Scotland, 2006 (EPS-AG, Edinburgh, Scotland, 2006), p. 1789.

[16] M. J. Barnes et al., Report No. CERN-2006-011, 2006.

[17] S. Gilardoni et al, in Proceedings of the 23rd Particle Accelerator Conference, Vancouver, Canada, 2009 (IEEE, Piscataway, NJ, 2009), p. 1581.

[18] S. Gilardoni et al., in Proceedings of the 23rd Particle Accelerator Conference, Vancouver, Canada, 2009 (IEEE, Piscataway, NJ, 2009), p. 1578.

[19] E. Benedetto et al., in Proceedings of the International Particle Accelerator Conference, IPAC-2010, Kyoto, Japan (ICR, Kyoto, 2010), p. 3619.

[20] H. Bartosik, D. Bodart, J. Borburgh, R. Brown, S. Damjanovic, S. Gilardoni, M. Giovannozzi, B. Goddard, C. Hernalsteens, M. Hourican, and M. Widorski, in Proceedings of the 3rd International Particle Accelerator Conference, IPAC-2012, New Orleans, LA, 2012 (IEEE, Piscataway, NJ, 2012), p. 499.

[21] S. Gilardoni, M. Giovannozzi, C. Hernalsteens, A. Lachaize, and G. Métral, in Proceedings of the 3rd International Particle Accelerator Conference, IPAC-2012, New Orleans, LA, 2012 (IEEE, Piscataway, NJ, 2012), p. 502.

[22] C. Hernalsteens, H. Bartosik, L. Drosdal, S. Gilardoni, M. Giovannozzi, A. Lachaize, Y. Papaphilippou, and A. Ulsrod, in Proceedings of the 4th International Particle Accelerator Conference, IPAC-2013, Shanghai, China, 2013 (JACoW, Shanghai, China, 2013), p. 2633.

[23] C. Hernalsteens, S. Damjanovic, S. Gilardoni, and M. Giovannozzi, in Proceedings of the 4th International Particle Accelerator Conference, IPAC-2013, Shanghai, China, 2013 (JACoW, Shanghai, China, 2013), p. 2636.

[24] C. Bertone et al., Report No. CERN-ACC-2014-0043, 2014.

[25] J. Borburgh, S. Damjanovic, S. Gilardoni, M. Giovannozzi, C. Hernalsteens, M. Hourican, A. Huschauer, K. Kahle, G. Le Godec, O. Michels, and G. Sterbini, First implementation of transversely split proton beams in the CERN Proton Synchrotron for the fixed-target physics programme, Europhys. Lett. 113, 34001 (2016).

[26] A. Huschauer, J. Borburgh, S. Damjanovic, S. Gilardoni, M. Giovannozzi, M. Hourican, K. Kahle, G. Le Godec, O. Michels, G. Sterbini, and C. Hernalsteens, Report No. CERN-ACC-2016-0100, 2016.

[27] C. Bovet and K. H. Reich, in 6th International Conference on High-Energy Accelerators, Cambridge, MA, USA, 1967, edited by R. A. Mack (Cambridge Electron Accelerator, Cambridge, 1967), p. 315.

[28] L. Arnaudon, Report No. CERN-AB-2006-084, 2006.

[29] H. Damerau, A. Funken, R. Garoby, S. Gilardoni, B. Goddard, K. Hanke, A. Lombardi, D. Manglunki, M. Meddahi, B. Mikulec, G. Rumolo, E. Shaposhnikova, M. Vretenar, and J. Coupard, Report No. CERN-ACC2014-0337, 2014.

[30] B. Mikulec, A. Findlay, V. Raginel, G. Rumolo, and G. Sterbini, in The 52nd ICFA Advanced Beam Dynamics Workshop on High-Intensity and High-Brightness Hadron Beams, HB2012, edited by N. Zhao, J. Chrin, D. Ji, 
C. Petit-Jean-Genaz, V. R. W. Schaa, and H. Yan (JACoW, Beijing, China, 2012), p. 175.

[31] E. Regenstreif, Report No. CERN 59-29, 1959.

[32] J.-P. Burnet et al., Report No. CERN-2011-004, 2011.

[33] A. Bazzani, G. Servizi, E. Todesco, and G. Turchetti, CERN Yellow Report No. 94-02, 1994.

[34] G. Sterbini, B. Dehning, S. S. Gilardoni, and A. Guerrero, in Fourth International Particle Accelerator Conference, IPAC-2014, edited by C. Petit-Jean-Genaz, G. Arduini, P. Michel, and R. Schaa (JACoW, Dresden, Germany, 2014), p. 3674.

[35] M. Juchno (private communication).

[36] N. A. Tahir, R. Schmidt, M. Brugger, R. Assmann, A. V. Shutov, I. V. Lomonosov, A. R. Piriz, D. H. H. Hoffmann, C. Deutsch, and V.E. Fortov, The CERN Super Proton
Synchrotron as a tool to study high energy density physics, New J. Phys. 10, 073028 (2008).

[37] A. Caldwell, E. Gschwendtner, K. Lotov, P. Muggli, and M. Wing, Report No. CERN-SPSC-2013-013, 2013.

[38] E. Gschwendtner, C. Bracco, B. Goddard, M. Meddahi, A. Pardons, E. Shaposhnikova, H. Timko, F. Velotti, and H. Vincke, in Proceedings of the 4th International Particle Accelerator Conference, IPAC-2013, Shanghai, China, 2013 (JACoW, Shanghai, China, 2013), p. 1253.

[39] (SHiP Collaboration), Report No. CERN-SPSC-2015-016, 2015; arXiv: 1504.04956.

[40] J. B. Adams, Report No. CERN-SPC-299, 1970.

[41] L. R. Evans, Report No. CERN-SPS-DI-MST-80-2, 1980.

[42] J. Bellemann (private communication). 